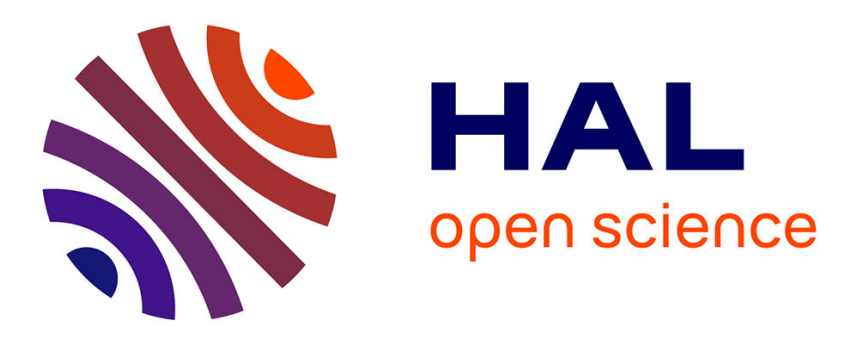

\title{
Three-dimensional instabilities and optimal perturbations of a counter-rotating vortex pair in stratified flows
}

Sabine Ortiz, Claire Donnadieu, Jean-Marc Chomaz

\section{To cite this version:}

Sabine Ortiz, Claire Donnadieu, Jean-Marc Chomaz. Three-dimensional instabilities and optimal perturbations of a counter-rotating vortex pair in stratified flows. Physics of Fluids, 2015, 27 (10), pp.106603. 10.1063/1.4934350 . hal-01234022

\section{HAL Id: hal-01234022}

\section{https://hal-ensta-paris.archives-ouvertes.fr/hal-01234022}

Submitted on 26 Nov 2015

HAL is a multi-disciplinary open access archive for the deposit and dissemination of scientific research documents, whether they are published or not. The documents may come from teaching and research institutions in France or abroad, or from public or private research centers.
L'archive ouverte pluridisciplinaire HAL, est destinée au dépôt et à la diffusion de documents scientifiques de niveau recherche, publiés ou non, émanant des établissements d'enseignement et de recherche français ou étrangers, des laboratoires publics ou privés. 


\section{AIP $\left.\right|_{\text {flyides of }} ^{\text {Phy }}$ Fluids}

\section{Three-dimensional instabilities and optimal perturbations of a counter- rotating vortex pair in stratified flows}

Sabine Ortiz, Claire Donnadieu, and Jean-Marc Chomaz

Citation: Physics of Fluids 27, 106603 (2015); doi: 10.1063/1.4934350

View online: http://dx.doi.org/10.1063/1.4934350

View Table of Contents: http://scitation.aip.org/content/aip/journal/pof2/27/10?ver=pdfcov

Published by the AIP Publishing

\section{Articles you may be interested in}

Numerical simulations of the near wake of a sphere moving in a steady, horizontal motion through a linearly stratified fluid at $\operatorname{Re}=1000$

Phys. Fluids 27, 035113 (2015); 10.1063/1.4915139

Vortex bursting and tracer transport of a counter-rotating vortex pair

Phys. Fluids 24, 025104 (2012); 10.1063/1.3684990

Three-dimensionalization of the stratified mixing layer at high Reynolds number

Phys. Fluids 23, 111701 (2011); 10.1063/1.3651269

Three-dimensional instabilities and transient growth of a counter-rotating vortex pair

Phys. Fluids 21, 094102 (2009); 10.1063/1.3220173

Elliptical-inertial instability of rotating Karman vortex streets

Phys. Fluids 17, 066602 (2005); 10.1063/1.1937348

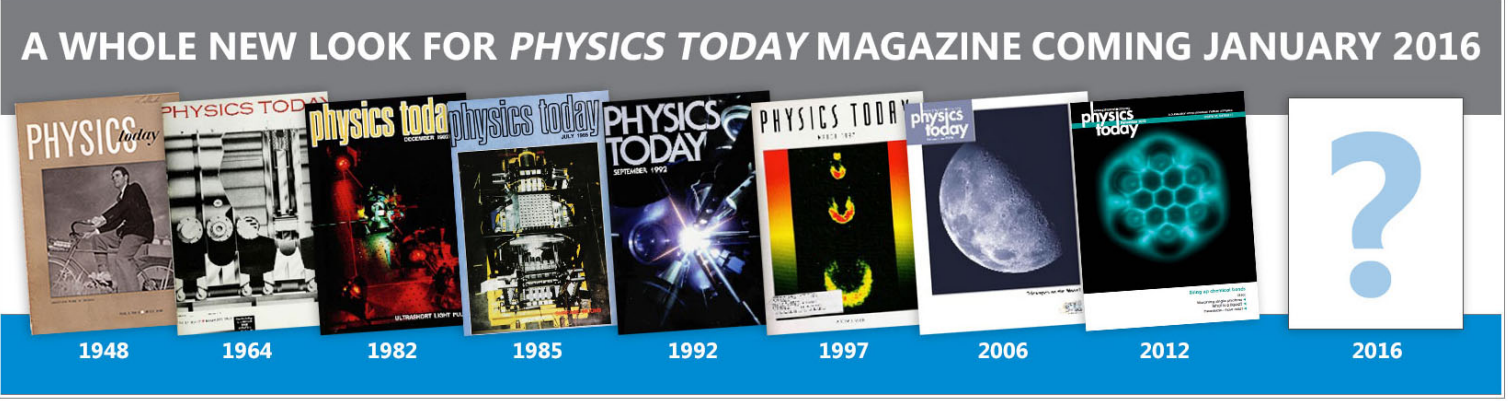




\title{
Three-dimensional instabilities and optimal perturbations of a counter-rotating vortex pair in stratified flows
}

\author{
Sabine Ortiz, ${ }^{1,2}$ Claire Donnadieu, ${ }^{1}$ and Jean-Marc Chomaz ${ }^{1}$ \\ ${ }^{1}$ LadHyX, École Polytechnique, Palaiseau Cedex 91128, France \\ ${ }^{2} U M E / D F A, E N S T A$, Chemin de la Hunière, 91761 Palaiseau Cedex, France
}

(Received 21 April 2015; accepted 5 October 2015; published online 27 October 2015)

This paper investigates the three-dimensional instabilities and the optimal perturbations on a pair of horizontal counter-rotating Lamb-Oseen vortices in a vertically stably stratified flow. Two-dimensional (2D) simulations are first performed, showing that while the dipole moves vertically against the stratification the vortex parameters: the radius $a^{*}$, the separation distance $b^{*}$, and the circulation $\Gamma^{*}$ are solely function of the time rescaled by the Brunt-Väisälä frequency $N$, independently of the Froude number, when the Reynolds number is large enough. Here, the Froude number is $F r=W_{0} / N b_{0}$ with $W_{0}$ the initial advection velocity of the dipole and $b_{0}$ the initial separation distance between the two vortices. For weak and moderate stratifications (large $F r$ ), the stratification acts on a long time scale compared to the advection time of the dipole implying that the 2D flow can be considered as quasi-steady. In that case, when three dimensional instabilities are added, a linear stability analysis of this $2 \mathrm{D}$ flow at different instants retrieves the instability peaks corresponding to the Crow instability for the long wavelengths and to the elliptic instability for the short wavelengths showing that the dynamics is almost unaffected by buoyancy effects. The Crow and elliptic instabilities scale with the instantaneous dipole parameters showing in particular that stratification promotes instability by reducing the distance $b^{*}$ between vortices [K. K. Nomura et al., "Short-wavelength instability and decay of a vortex pair in stratified fluid," J. Fluid Mech. 553, 283-322 (2006)]. For strong stratifications (Froude numbers of order unity or smaller), the quasi-steady approximation is not valid, and the question of stability should be formulated in a different way, by, for example, searching for the transient growth of the energy of perturbation that may be computed for steady or unsteady base flow. Then, for each time horizon $\tau$, we should determine the critical perturbation leading to the largest energy growth by the time $\tau$. Presently, we compute the optimal perturbations at two time horizons $\tau=4$ and $\tau=10$ dimensionalized by $2 \pi b_{0}^{2} / \Gamma_{0}$ with a direct-adjoint technique which takes into account the evolution of the base flow. In the homogeneous case, this technique allows to investigate the effect of the weak unsteadiness of the flow due to viscous diffusion which induces a growth of the vortex core radius $a^{*}$. Both Crow and elliptic instabilities are retrieved in the optimal response and in the energy gain curves. Even if very slow, the viscous diffusion is found to increase the gain of the antisymmetric elliptic perturbation compared to the symmetric one. When the fluid is stratified, peaks at small wavenumber and at wavenumber of the order of the vortex core size are found for all Froude numbers with optimal responses strongly resembling, respectively, the Crow and the elliptic modes with optimal gains corresponding to mean growth rates larger than in the homogeneous case for both modes. However, as the strength of stratification increases (Froude numbers smaller than 2), optimal perturbations start departing from their homogeneous counterpart with large perturbation in the wake of the dipole associated with density effects. (C) 2015 AIP Publishing LLC. [http://dx.doi.org/10.1063/1.4934350] 


\section{INTRODUCTION}

The wake which forms behind an aircraft is a pair of horizontal counter-rotating vortices propagating downwards. Depending on the atmospheric conditions, such dipole can persist over a long time or be rapidly destroyed. The dynamics of such dipoles has been widely studied in the past and recent years. In homogeneous fluids, a vortex pair is unstable with respect to three-dimensional perturbations. Crow ${ }^{1}$ has discovered a long-wavelength instability, symmetric with respect to the plane separating the two vortices. The existence of a short wavelength elliptic instability has been discussed by Moore and Saffman, ${ }^{2}$ Tsai and Widnall ${ }^{3}$ and numerous subsequent articles for both symmetric and antisymmetric modes. Physically, the Crow instability comes from the coupling through mutual induction of the slow $m=1$ Kelvin mode, traveling on the two vortices, whereas the elliptic instability may be viewed as the resonant interaction of the fast Kelvin mode $m=-1$ and $m=1$ traveling on the same vortex coupled by the steady strain imposed by the other vortex. However, in many atmospheric situations, this dipole propagating downwards evolves under the influence of the stable stratification of the atmosphere. Investigations of the two-dimensional (2D) evolution of a counter-rotating vortex pair in a stably stratified flow have shown a reduction of the separation distance between the vortices with acceleration of the dipole under the effect of the baroclinic torque of the displaced fluid (Scorer and Davenport, ${ }^{4}$ Hill, ${ }^{5}$ Spalart, ${ }^{6}$ Garten et al. ${ }^{7}$ ). The three-dimensional dynamics of a vortex pair in stratified flow has been observed experimentally by Sarpkaya ${ }^{8}$ and Delisi and Robins ${ }^{9}$ and numerically by Robins and Delisi ${ }^{10}$ and Garten et al. ${ }^{11}$ who all showed a reduction of separation distance and deceleration. Nomura et al., ${ }^{12}$ in their three-dimensional direct numerical simulations, observe a decrease of the separation distance with a nearly constant descent speed for weakly stratified flows and a deceleration for moderate and strong stratifications. The influence of the stable stratification on the three-dimensional instabilities has received much less attention. Robins and Delisi ${ }^{10}$ investigate the effect of stratification on the Crow instability and observe that the vortices eventually link and form rings, more rapidly as stratification increases. For stronger stratification, they observe the formation of three-dimensional structures that they called puffs. Garten et al. ${ }^{11}$ observe that for relatively strong stratification, the Crow instability grows faster since density effects push the vortices closer. Direct numerical simulations of Nomura et al. ${ }^{12}$ on the short-wavelength instability of a counter-rotating vortex pair in presence of stable stratification have suggested that, for weak and moderate stratifications, the instability mechanism is not modified by the stratification and corresponds to the elliptic instability as in homogeneous media. The instability appears earlier than in the unstratified case, owing to the decrease of the separation distance between the vortices as they propagate downwards, decrease that induces larger ellipticity of the vortices and thus enhances the instability. For strongly stratified flows, the instability cannot be considered elliptic anymore since the primary vortices are strongly deformed by the stratification.

In the present paper, the stability of a stratified wake is addressed using both linear stability analysis in a quasi-steady approximation and exact optimal perturbation computation. The two-dimensional evolution of a Lamb-Oseen vortex pair in stably stratified fluid is documented in Sec. II. In the case of weak stratification, the evolution of the two-dimensional flow due to stratification is slow and can be neglected at leading order (quasi-steady approximation), which justifies the use of linear stability analysis on the frozen flow field at different instants (Sec. III). In the case of strong stratification, stability analysis of a frozen state is no longer legitimate and the development of perturbations is addressed by computing transient growth of energy via an optimization procedure. The optimal perturbations are computed at different time horizons, with a direct-adjoint technique similar to the one used in the steady case (Donnadieu et al. ${ }^{13}$ ) but taking into account the evolution of the flow. Those results are presented in Sec. IV.

\section{TWO-DIMENSIONAL SIMULATIONS OF COUNTER-ROTATING VORTICES IN STRATIFIED FLUID}

\section{A. Equations and numerical method}

We first investigate the two-dimensional evolution of a pair of counter-rotating vortices in a stable vertical stratification. The initial state is the superposition of two circular Lamb-Oseen 
vortices of initial circulation $\Gamma_{0}$, initial radius $a_{0}$, and initial separation distance $b_{0}$ with opposite signs vorticity corresponding to the dimensional initial vorticity,

$\omega_{b y}^{*}\left(x^{*}, z^{*}, t^{*}=0\right)=\frac{\Gamma_{0}}{\pi a_{0}^{2}} \exp \left(-\frac{\left(x^{*}-x_{1}^{*}\right)^{2}+\left(z^{*}-z_{1}^{*}\right)^{2}}{a_{0}^{2}}\right)-\frac{\Gamma_{0}}{\pi a_{0}^{2}} \exp \left(-\frac{\left(x^{*}-x_{2}^{*}\right)^{2}+\left(z^{*}-z_{2}^{*}\right)^{2}}{a_{0}^{2}}\right)$,

where $\omega_{b y}^{*}$ is the axial vorticity, $\left(x^{*}, z^{*}\right)$ corresponds, respectively, to the Cartesian transverse and vertical dimensional quantities, and $\left(x_{1}^{*}, z_{1}^{*}\right)$ and $\left(x_{2}^{*}, z_{2}^{*}\right)$ are the initial coordinates of the two vortex centroids. The $\Gamma_{0}, a_{0}$, and $b_{0}$ denote dimensional circulation, radius, and distance separation $\left(b_{0}=\sqrt{\left(x_{2}^{*}-x_{1}^{*}\right)^{2}+\left(z_{2}^{*}-z_{1}^{*}\right)^{2}}\right.$ of vortices. The total dimensional density $\rho_{t}^{*}$ and pressure fields $p_{t}^{*}$ are written as

$$
\begin{aligned}
& \rho_{t}^{*}\left(x^{*}, z^{*}, t^{*}\right)=\rho_{0}+\frac{\partial \bar{\rho}^{*}}{\partial z^{*}} z^{*}+\rho^{*}\left(x^{*}, z^{*}, t^{*}\right), \\
& p_{t}^{*}\left(x^{*}, z^{*}, t^{*}\right)=p_{0}+\bar{p}^{*}\left(z^{*}\right)+p^{*}\left(x^{*}, z^{*}, t^{*}\right),
\end{aligned}
$$

where $\rho_{0}$ and $p_{0}$ are constant reference values, $\frac{\partial \bar{\rho}^{*}}{\partial z^{*}} z^{*}$ is a stable linear density profile with $\frac{\partial \bar{\rho}^{*}}{\partial z^{*}}$ constant and negative, $\bar{p}^{*}\left(z^{*}\right)$ is the corresponding hydrostatically balanced pressure field, and $\rho^{*}$ and $p^{*}$ are the density and pressure perturbations which are assumed to be zero at the initial instant. The evolution of the velocity, vorticity, pressure, and density perturbation $\left[\mathbf{u}^{*}, \omega^{*}, p^{*}, \rho^{*}\right]\left(x^{*}, z^{*}, t^{*}\right)$ is governed by the following two-dimensional Navier-Stokes equations under the Boussinesq approximation:

$$
\left\{\begin{array}{l}
\frac{\partial \mathbf{u}^{*}}{\partial t^{*}}=\mathbf{u}^{*} \times \omega^{*}-\nabla\left(\frac{p^{*}}{\rho_{0}}+\frac{\mathbf{u}^{* 2}}{2}\right)+v \Delta \mathbf{u}^{*}-\frac{\rho^{*}}{\rho_{0}} g \mathbf{e}_{\mathbf{z}}, \\
\nabla \cdot \mathbf{u}^{*}=0, \\
\frac{\partial \rho^{*}}{\partial t^{*}}=-\mathbf{u}^{*} \cdot \nabla \rho^{*}-\frac{\partial \bar{\rho}^{*}}{\partial z^{*}} u_{z}^{*}+\kappa \Delta \rho^{*},
\end{array}\right.
$$

where $v$ is the kinematic viscosity of the flow, $g$ is the gravity, and $\kappa$ is the diffusivity of the stratifying agent (salt or temperature). The characteristic length scale is the initial vortex separation distance between the two vortices $b_{0}$, the velocity scale is the initial advection velocity of the dipole $W_{0}=\Gamma_{0} / 2 \pi b_{0}$; hence, the characteristic time scale is the dipole advection time scale $T_{A}=2 \pi b_{0}^{2} / \Gamma_{0}$ and $b_{0} \rho_{0} N^{2} / g$ is the density scale with $N=\sqrt{-\left(g / \rho_{0}\right) d \bar{\rho}^{*} / d z^{*}}$ the Brunt-Väisälä frequency. With this choice of scales, the Navier Stokes equations for the non-dimensionalized quantities $[\mathbf{u}, \omega, p, \rho](x, z, t)$ become

$$
\left\{\begin{array}{l}
\frac{\partial \mathbf{u}}{\partial t}=\mathbf{u} \times \omega-\nabla \cdot\left(p+\frac{\mathbf{u}^{2}}{2}\right)+\frac{1}{R e} \Delta \mathbf{u}-F r^{-2} \rho \mathbf{e}_{\mathbf{z}}, \\
\nabla \cdot \mathbf{u}=0 \\
\frac{\partial \rho}{\partial t}=-\mathbf{u} \cdot \nabla \rho+u_{z}+R e^{-1} S c^{-1} \Delta \rho,
\end{array}\right.
$$

where $S c=v / \kappa$ is the Schmidt number, here taken equal to unity, $F r=W_{0} / N b_{0}$ is the Froude number and $R e=W_{0} b_{0} / v$ is the Reynolds number. We also define a second Reynolds number based on the initial circulation of the vortices as follows:

$$
R e_{\Gamma_{0}}=\frac{\Gamma_{0}}{v}=\frac{2 \pi W_{0} b_{0}}{v}=2 \pi R e .
$$

Two-dimensional Navier-Stokes equations (5) are numerically integrated using a pseudospectral method in Cartesian coordinates with periodic boundary conditions as described in Delbende et al. ${ }^{14}$ With the application of the two-dimensional Fourier transform on the velocity, vorticity, pressure, and density perturbation fields,

$$
[\mathbf{u}, \omega, p, \rho](x, z, t)=\iint[\hat{\mathbf{u}}, \hat{\omega}, \hat{p}, \hat{\rho}]\left(k_{x}, k_{z}, t\right) e^{i\left(k_{x} x+k_{z} z\right)} d k_{x} d k_{z},
$$


where $k_{x}$ and $k_{z}$ are the transverse and vertical components of the wavevector $\mathbf{k}_{2 D}$, Navier-Stokes equations (5) become in the spectral space,

$$
\left\{\begin{array}{l}
\frac{\partial \hat{\mathbf{u}}}{\partial t}=\mathbf{P}\left(\mathbf{k}_{2 \mathbf{D}}\right)\left[\widehat{\mathbf{u} \times \boldsymbol{\omega}}-\hat{\rho} F r^{-2} \mathbf{e}_{\mathbf{z}}\right]-\operatorname{Re}^{-1}\left|\mathbf{k}_{2 D}\right|^{2} \hat{\mathbf{u}}, \\
\frac{\partial \hat{\rho}}{\partial t}=-i \mathbf{k} \cdot \widehat{\mathbf{u} \rho}+\hat{u}_{z}-\operatorname{Re}^{-1} S c^{-1}\left|\mathbf{k}_{2 D}\right|^{2} \hat{\rho},
\end{array}\right.
$$

where $\mathbf{P}\left(\mathbf{k}_{2 D}\right)$ is the projection operator on the space of divergence-free fields which, in Fourier space, may be expressed as a tensor with components $P_{i j}=\delta_{i j}-k_{i} k_{j} / \mathbf{k}_{2 D}^{2}$. In this pseudo-spectral method, the nonlinear terms $\mathbf{u} \times \omega$ and $\mathbf{u} \rho$ are evaluated in the physical space. Time integration of inviscid terms is done using a second-order Adams-Bashforth scheme whereas dissipative terms $R e^{-1} \Delta \mathbf{u}$ and $R e^{-1} S c^{-1} \Delta \rho$ are integrated exactly in the Fourier space.

The time step is set to $\Delta t=10^{-3}$. The size of the periodic box is $L_{x}=12$ in the transverse direction and $L_{z}=24$ in the vertical direction with $512 \times 1024$ collocation points equally spaced in the $x$ and $z$ directions. The transverse and vertical lengths are sufficient to minimize the effects of periodic boundary conditions $\left(L_{x}=60 a_{0} / b_{0}\right)$ to prevent the dipole from encountering its wake on its downward descent during the time interval considered here. The spatial discretisation allows approximately 8 collocation points along a vortex core diameter.

\section{B. The two-dimensional evolution of the flow}

The Reynolds number is $R e_{\Gamma_{0}}=2400$ and the Froude numbers considered are $\mathrm{Fr}=\infty$ (homogeneous flow), 10, 5, 2, and 1 . The initial aspect ratio of the dipole is $a_{0} / b_{0}=0.2$. As the counterrotating vortices propagate downwards, they evolve under the influence of the stratification.

Figs. 1 and 2 represent the evolution of the axial vorticity $\omega_{y}$ of the vortex pair and the density perturbation $\rho$ at two instants rescaled by the Brunt-Väisälä frequency $N t^{*}=1$ and $N t^{*}=2$ for Froude numbers 10, 5, 2, and 1 (equivalent to rescale the non-dimensionalized time $t$ by the Froude number $\mathrm{Fr}: \mathrm{Nt}^{*}=\mathrm{Fr}^{-1} t$ ).

As the vortices propagate downwards, density perturbations (right column of Figs. 1 and 2) appear at the instantaneous location of the dipole and the isodensity lines, initially horizontal (not shown here), are strongly deformed: the low density fluid located at the initial altitude of the dipole is entrained inside the vortex cores. As the dipole descends, this light fluid is brought to heavier density regions and a strong density variation forms at the boundary of the dipole: intense horizontal density gradients appear around and in the lee of the vortex pair.

The larger the Froude number, the longer the density wake behind the dipole at a given $N t^{*}=F r^{-1} t$. In Figs. 1 and 2, we observe two distinct structures of the density perturbation inside the core. Either iso-density contours follow iso-vorticity contours (large $\mathrm{Fr}$ ) or iso-density contours are horizontal (small $F r$ ). This may be interpreted as the results of two effects, the transport of the density by the vortices, and the uniformization of the density on closed trajectories in the cores of the vortices. Since the snapshots of the flow have been taken at $N t^{*}=j(j=1,2)$, the non-dimensional distance travelled by the dipole is of order $j F r$ leading to a perturbation density due to the transport proportional to $j F r$ yielding a gradient with the outside of the core also proportional to $j F r$. At the same time, the gradient of the total density inside the vortices should be close to uniform; hence, the gradient of $\rho$ should compensate the base stratification. This gradient is of order -1 with the present non-dimensionalisation. Therefore, the two behaviors observed are the competition between the gradient between the core of the vortices and the outside of order $j F r$ and the gradient inside the vortices of order -1 .

As shown by Garten et al. ${ }^{7}$ for high Froude numbers, i.e., for flows dominated by advection, we observe on the left column of Figs. 1 and 2 that, as the dipole propagates downwards, opposite-sign vorticity is created around and behind the primary vortices through the baroclinic torque which pushes the vortices towards one another, hence reducing the separation distance $b^{*}$ between the vortices. This secondary vorticity is detrained during the descent of the dipole as observed in Figs. 2(a), 2(c), and 2(e). 


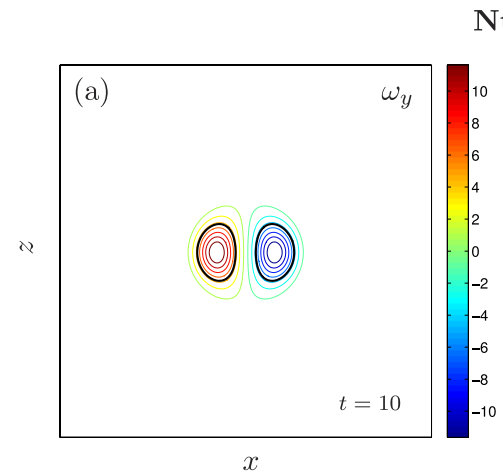

$\mathrm{Nt}^{*}=1$
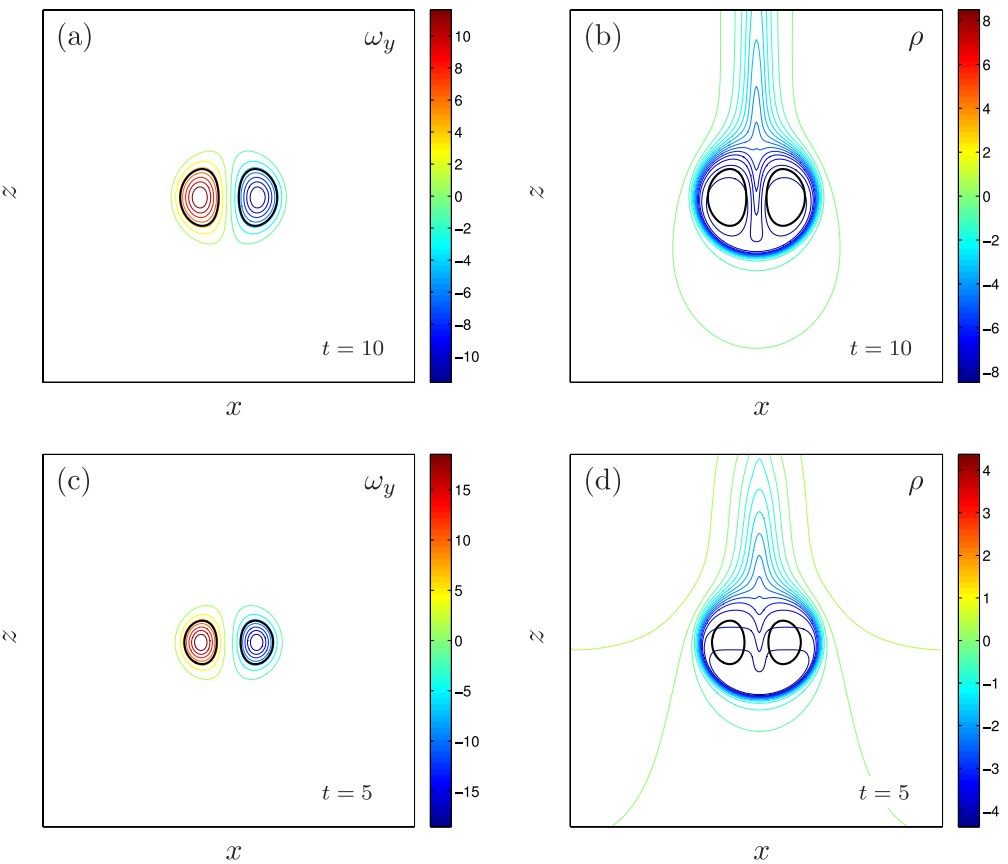

$$
F r=10
$$
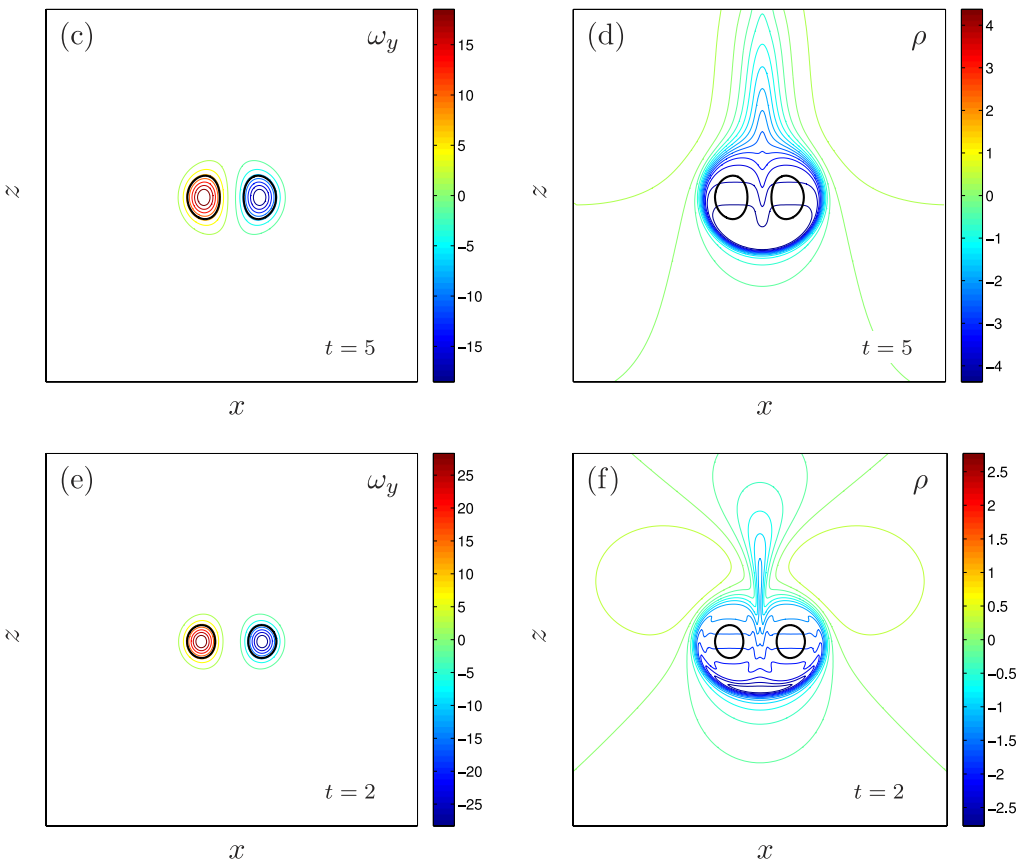

$$
F r=2
$$

$$
\mathrm{Fr}=5
$$
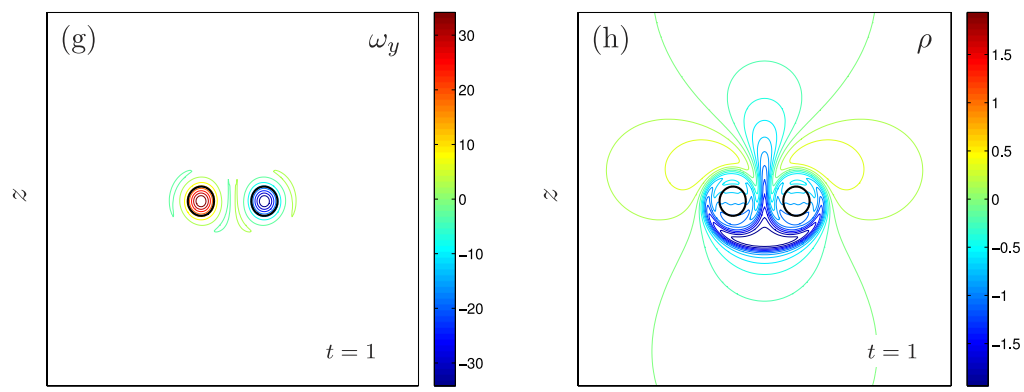

$$
F r=1
$$

FIG. 1. 2D flow at $N t^{*}=F r^{-1} t=1$-isovalues of the (a), (c), (e), and (g) axial vorticity $\omega_{y}$ and (b), (d), (f), and (h) density $\rho$ of the vortex pair in the $(x, z)$ plane at $F r^{-1} t=N t^{*}=1$ for (a) and (b) $F r=10$, (c) and (d) $F r=5$, (e) and (f) $F r=2$, and (g) and (h) $F r=1$. The heavy black lines correspond to the isocontours $\omega_{y} / \omega_{y}^{\max }= \pm \exp (-1)$. The size of the domain shown is $6 b_{0} \times 6 b_{0}$ whereas the computation domain is much larger $12 b_{0} \times 24 b_{0}$, where $b_{0}$ is the initial separation distance between the vortices.

In the presence of stratification, the non-dimensional circulation $\Gamma=\Gamma^{*} / \Gamma_{0}$, the non-dimensional separation distance between the vortices $b=b^{*} / b_{0}$, and the non-dimensional radius $a=a^{*} / b_{0}$ evolve as a function of time as plotted in Fig. 3. The vortex radius $a^{*}$ is determined using the vorticity polar moment like in Donnadieu et al.: ${ }^{13} a^{2}=\left\langle\left[\left(x-x_{2}\right)^{2}+\left(z-z_{2}\right)^{2}\right] \omega_{B y}\right\rangle /\left\langle\omega_{B y}\right\rangle$ with $\langle$. denoting the integration over the semi-infinite domain $x>0$. The distance between the two vortices 


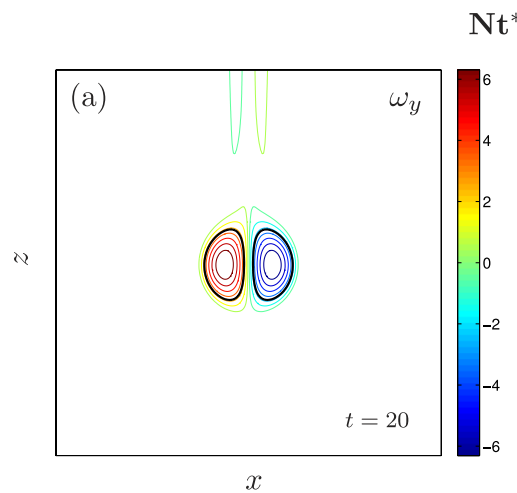

$\mathrm{Nt}^{*}=\mathbf{2}$
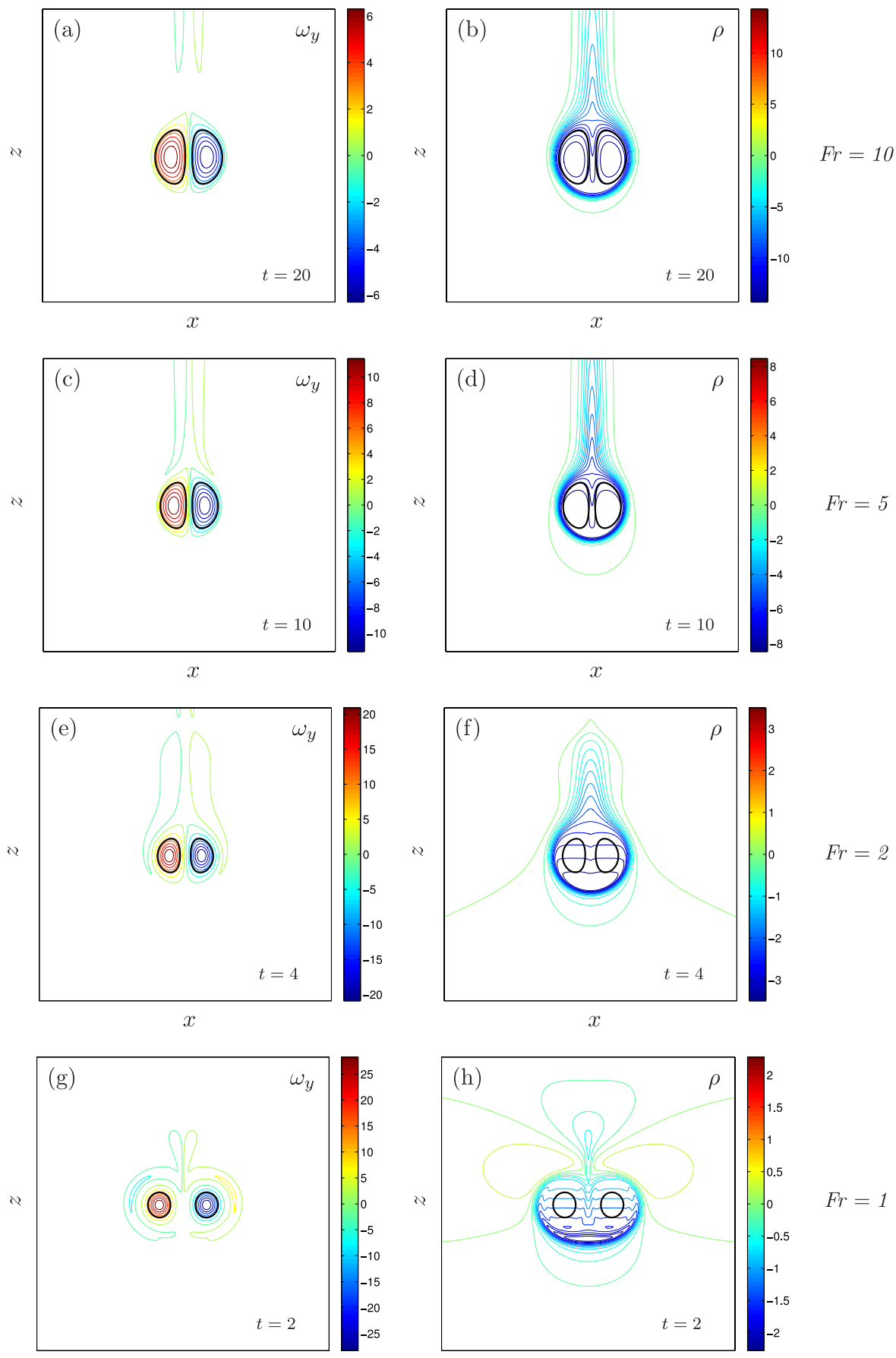

$$
F r=2
$$

$$
F r=1
$$

FIG. 2. 2D flow at $\mathrm{Nt}^{*}=\mathrm{Fr}^{-1} \mathrm{t}=2$-same legend as Fig. 1 except that $\mathrm{Fr}^{-1} t=N t^{*}=2$.

is $b=\left|x_{2}-x_{1}\right|,\left(x_{1}, z_{1}\right)$, and $\left(x_{2}, z_{2}\right)$ being the locations of the vorticity extrema and $z_{2}=z_{1}$. The circulation of the vortices is determined on one primary vortex computing the flux of vorticity through the surface limited by the contour defining its core.

We observe in Fig. 3(a) a growth of $a$ as function of time solely due to viscous diffusion for $F r=\infty$, and in good agreement with the theoretical prediction of Batchelor ${ }^{15}$ represented with a dashed line in Fig. 3(a), 


\section{(a)}

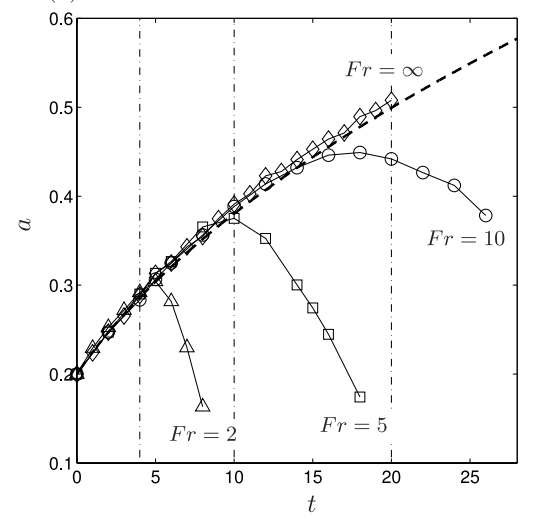

(b)

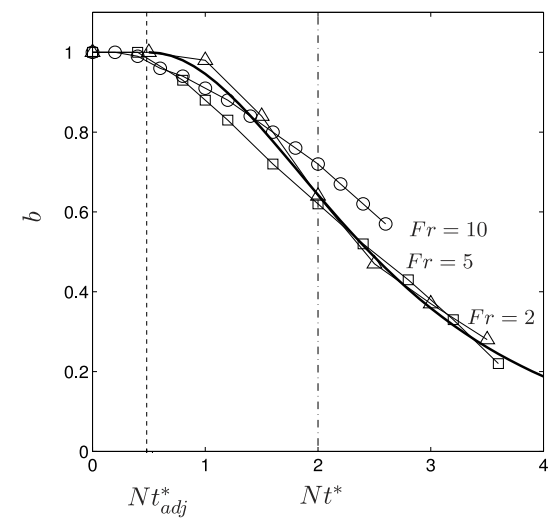

FIG. 3. Temporal evolution of the dipole parameters-evolution of (a) the radius $a$ of the vortex core as function of time $t$ and (b) the separation distance $b$ between the vortices as function of time rescaled by the Froude number $\mathrm{Fr}^{-1} t=N t^{*}$ for $F r=\infty(\diamond), 10(\circ), 5(\square)$, and $2(\triangle)$. The dashed-dotted lines are the instant $F r^{-1} t=N t^{*}=2$. The dashed line in (a) corresponds to the diffusion law (Batchelor ${ }^{15}$ ) (9), valid for $F r=\infty$. The continuous line in (b) represents the prediction of $\operatorname{Saffman}^{16}(10)$ for the effect of weak stratification in an inviscid fluid after rescaling by $N\left(t^{*}-t_{a d j}^{*}\right)$, plotted between $N t_{a d j}^{*}$ and $N t^{*}=4$, with $t_{a d j}^{*}$ the time from which the distance $b(t)$ starts to decrease.

$$
a(t)=\sqrt{a_{0}^{2} / b_{0}^{2}+4 t / R e}
$$

For the stratified cases, the radius of the vortices $a$ grows by viscous diffusion until $t^{*} \simeq 2 / N$ (or $t=2 F r$ ) indicated by vertical dashed-dotted lines and then decreases through interaction with opposite sign vorticity generated by the baroclinic torque.

In Fig. 3(b), the non-dimensional separation distance $b$ between the vortices is plotted versus $N t^{*}=F r^{-1} t$. In the unstratified case $F r=\infty$ (data computed but not presented in Fig. 3(b) since $N=0$ ), the distance $b$ remains constant during the descent of the dipole. In the stratified cases, the distance $b$ decreases after a time $N t_{a d j}^{*}$ (with $t_{a d j}^{*}$ the dimensional adjustment time beyond which the distance vortex separation starts to decrease) and Fig. 3 shows that its evolution is nearly independent of $\mathrm{Fr}$ when plotted as function of $N t^{*}=\mathrm{Fr}^{-1} t$. The evolution compares reasonably well with theoretical prediction of Saffman, ${ }^{16}$ retrieved by Spalart ${ }^{6}$ for a two-dimensional quasi-steady inviscid flow with weak stratification

$$
b(t)=\frac{1}{\cosh \left((2.85 / 2 \pi)^{1 / 2} N t^{*}\right)} .
$$

After rescaling by $N\left(t^{*}-t_{a d j}^{*}\right)$, we have plotted $b(t)$ function of $N t^{*}$ by a continuous line between $N t_{a d j}^{*}$ and $N t^{*}=4$ in Fig. 3(b). The vortices come into contact when the separation distance $b$ between the vortices becomes equal to twice the radius of one vortex $b=2 a$, which happens at $N t^{*}=$ $F r^{-1} t \simeq 2$ for $F r=2, N t^{*}=F r^{-1} t \simeq 1.6$ for $F r=5$, and $N t^{*}=F r^{-1} t \simeq 1.4$ for $F r=10$ when the viscous increase of $a$ with time is taken into account. These instants mark the departure of the evolution of $a$ from viscous law (9) and are close to the instant $N t^{*}=2$ marked by vertical dashed-dotted lines in Fig. 3(a). After this instant, the two vortices stay in contact and $a$ starts to decrease.

In unstratified fluids, $\Gamma$ is constant as time evolves whereas the circulation significantly decreases as function of time for stratified fluids as observed in Fig. 4(c) which represents the Reynolds number based on the circulation $R e_{\Gamma}=\Gamma / v$ as a function of $N t^{*}=F^{-1} t$. This decrease, which happens earlier with increasing stratification, is due to the combined effect of destruction of the primary vorticity due to baroclinic torque and to the diffusion of each vortex in the other after they come in contact, here after $N t^{*}=\mathrm{Fr}^{-1} t \simeq 2$.

Fig. 4(a) shows that $a / b$ increases with time since, as discussed previously, the radius $a$ of the vortices first increases by viscous diffusion and the separation distance between the vortices decreases due to the stratification. The strain $\Gamma / 2 \pi b^{2}$ is nearly constant and equal to 1 until $N t^{*}=\mathrm{Fr}^{-1} t=1$ for the three Froude numbers considered. For the weakly stratified flow $\mathrm{Fr}=10$, the strains remain close to 1 as time evolves suggesting that the strain is not significantly altered 
(a)

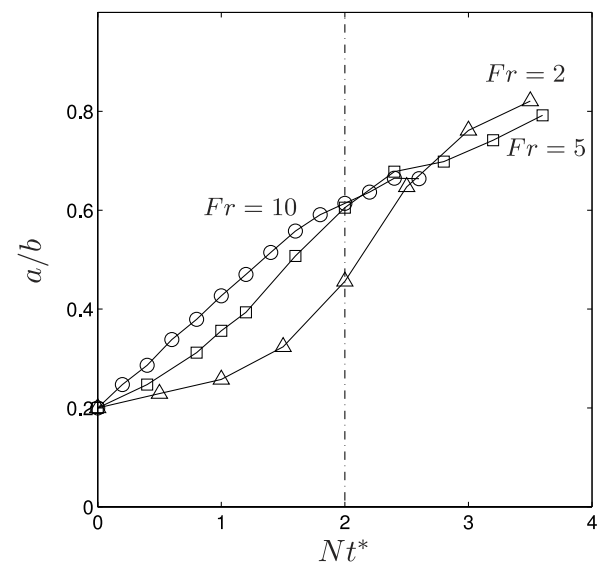

(b)

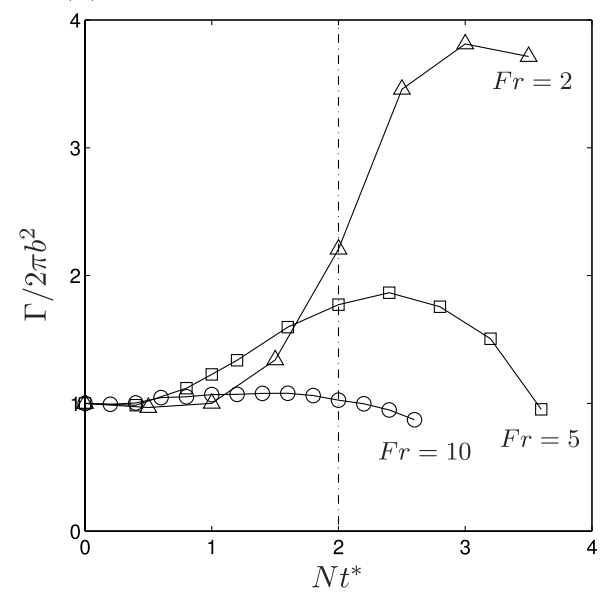

(c)

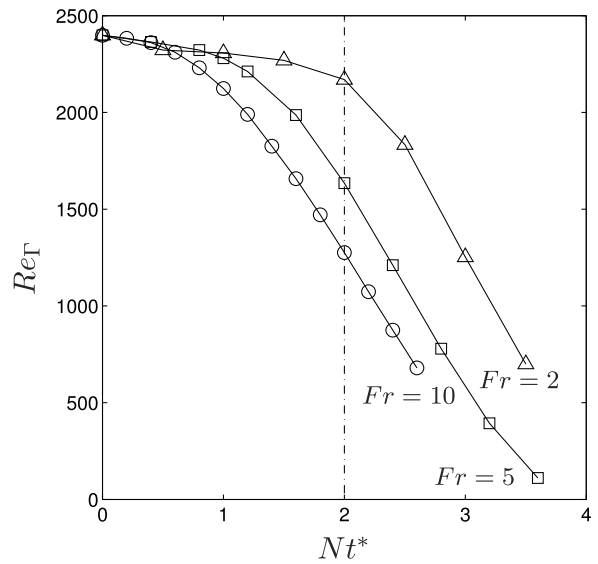

FIG. 4. Temporal evolution of the dipole parameters-evolution of (a) the aspect ratio $a / b$ of the dipole, (b) the nondimensional external strain rate $\Gamma / 2 \pi b^{2}$, and (c) the Reynolds number $R e_{\Gamma}$ as function of time rescaled by the Brunt-Väisälä frequency $\mathrm{Fr}^{-1} t=N t^{*}$ for $10(\circ), 5(\square)$, and $2(\triangle)$. Same legend as Fig. 3.

by the stratification. For the moderately stratified flows $F r=5$ and 2, the strain strongly increases between $N t^{*}=F r^{-1} t=1$ and $N t^{*}=F r^{-1} t=2.5$ for $F r=5$ and $N t^{*}=F r^{-1} t=3$ for $F r=2$, faster with increasing stratification.

The horizontal vortex dipole is characterized by a dipole aspect ratio $a / b$ and a Reynolds number $\operatorname{Re}_{\Gamma}$ (Figs. 3 and 4 ) which are not representative of aircraft trailing vortices (dipole aspect ratio 
much smaller $a / b$ of order 0.01 and greater Reynolds number, Delisi et $\left.a l .{ }^{17}\right)$. We address now the linear stability analysis of such counter-rotating vortices in a stratified fluid, in order to investigate the effect of the stratification on instability mechanisms compared with the unstratified case. ${ }^{13}$

\section{LINEAR STABILITY ANALYSIS IN STRATIFIED FLUID}

In the case of weak stratification, i.e., for large Froude numbers $F r$, the stratification acts on a long time scale $1 / N$ on the base velocity field compared to the advection time $2 \pi b^{* 2 / \Gamma^{*}}$ of the dipole, the base velocity field can therefore be considered as quasi-steady and a classical linear stability analysis describes the leading order dynamics of the perturbation driven by inertial mechanisms (hyperbolic, elliptic, or centrifugal instabilities) as discussed for the stratified mixing layer by Caulfield and Peltier. ${ }^{18}$ Even in the absence of stratification $(F r=\infty)$, the viscous diffusion is also a source of unsteadiness of the flow with time scale $a^{* 2} / v$ which may also be taken into account via the quasi-steady approximation when the Reynolds number is large enough.

\section{A. 2D base flow}

To perform the linear stability analysis, base flows are extracted from the two-dimensional computation presented in Sec. II at the two instants $\mathrm{Fr}^{-1} t=N t^{*}=1$ and $\mathrm{Fr}^{-1} t=N t^{*}=2$, with axial vorticity and density perturbation already shown in Figs. 1 and 2 . The flow can be considered quasi-steady only in the frame moving down with the dipole. But defining the advection velocity of this unsteady dipole is not unique since different portions of the flow (in particular the vortex cores and the wake) propagate at different speeds. Here, we chose to define the advection velocity as the velocity of the points of maximum vorticity at the center of the vortices. Sensitivity of the growth rate to the actual value of the advection velocity has been computed: a change of $5 \%$ of this velocity changes the growth rate by less than $4 \%$.

\section{B. Linearized equations}

Infinitesimal three-dimensional perturbations are superposed on this bidimensional base flow now assumed steady (the frozen base flow approximation). At leading order, the perturbations satisfy the linearized Navier-Stokes (LNS) equations in the Boussinesq approximation,

$$
\left\{\begin{array}{l}
\frac{\partial \mathbf{u}^{\prime}}{\partial t}=\mathbf{U}_{B} \times \omega^{\prime}+\mathbf{u}^{\prime} \times \mathbf{\Omega}_{B}-\nabla \cdot\left(p^{\prime}+\mathbf{u}^{\prime} \cdot \mathbf{U}_{B}\right)-\rho^{\prime} F r^{-2} \mathbf{e}_{\mathbf{z}}+R e^{-1} \Delta \mathbf{u}^{\prime}, \\
\nabla \cdot \mathbf{u}^{\prime}=0, \\
\frac{\partial \rho^{\prime}}{\partial t}=-\mathbf{U}_{B} \cdot \nabla \rho^{\prime}-\mathbf{u}^{\prime} \cdot \nabla \rho_{B}+u_{z}^{\prime}+R e^{-1} S c^{-1} \Delta \rho^{\prime},
\end{array}\right.
$$

where $\left[\mathbf{u}^{\prime}, \omega^{\prime}, \rho^{\prime}, p^{\prime}\right](x, y, z, t)$ are the velocity, the vorticity, the density, and the pressure of the three-dimensional perturbation and $\mathbf{U}_{B}=\left(u_{B}, 0, w_{B}\right)$ and $\mathbf{\Omega}_{B}=\left(0, \omega_{B}, 0\right)$ are the velocity and the vorticity of the base flow and $\rho_{B}$ the density perturbation associated with the base flow in the frame moving with the dipole. As the base state is uniform along the $y$ axis, the perturbations can be decomposed into normal modes,

$$
\left[\mathbf{u}^{\prime}, \omega^{\prime}, \rho^{\prime}, p^{\prime}\right](x, y, z, t)=[\tilde{\mathbf{u}}, \tilde{\omega}, \tilde{\rho}, \tilde{p}](x, z, t) \mathrm{e}^{i k_{y} y}+\text { c.c. },
$$

where $k_{y}$ is the axial wavenumber and c.c. denotes the complex conjugate.

\section{Numerical method}

Linearized Navier-Stokes equations (11) are integrated using the pseudo-spectral method in Cartesian coordinates with periodic boundary conditions similar to the one described for the base flow. The velocity, vorticity, density, and pressure of the normal mode corresponding to the axial 
wavenumber $k_{y}$ are expressed in Fourier space,

$$
[\tilde{\mathbf{u}}, \tilde{\boldsymbol{\omega}}, \tilde{\rho}, \tilde{p}](x, z, t)=\int[\hat{\mathbf{u}}, \hat{\omega}, \hat{\rho}, \hat{p}]\left(k_{x}, k_{z}, t\right) e^{i\left(k_{x} x+k_{z} z\right)} d k_{x} d k_{z} .
$$

In spectral space, linear Navier-Stokes equations (11) become

$$
\left\{\begin{array}{l}
\frac{\partial \hat{\mathbf{u}}}{\partial t}=\mathbf{P}(\mathbf{k})\left[\widehat{\mathbf{U}_{B} \times \tilde{\boldsymbol{\omega}}}+\widetilde{\mathbf{u} \times \mathbf{\Omega}_{B}}-\hat{\rho} F r^{-2} \mathbf{e}_{\mathbf{z}}\right]-R e^{-1} \mathbf{k}^{2} \hat{\mathbf{u}}, \\
\frac{\partial \hat{\rho}}{\partial t}=-i \mathbf{k} \cdot \widehat{\mathbf{U}_{B} \tilde{\rho}}-\widetilde{\mathbf{u}} \cdot \widehat{\nabla \rho_{B}}+\hat{u}_{z}-\operatorname{Re}^{-1} S c^{-1} \mathbf{k}^{2} \hat{\rho},
\end{array}\right.
$$

where $\mathbf{k}=\left(k_{x}, k_{y}, k_{z}\right)$ is the total wavevector and as in the $2 \mathrm{D}$ case, $\mathbf{P}(\mathbf{k})$ is the projection operator on the space of divergence-free fields: $P_{i j}=\delta_{i j}-k_{i} k_{j} / \mathbf{k}^{2}$. Time integration of inertial terms is done via a second-order Adams-Bashforth scheme whereas the dissipative terms $R^{-1} \mathbf{k}^{2} \hat{\mathbf{u}}$ and $R e^{-1} S c^{-1} \mathbf{k}^{2} \hat{\rho}$ are integrated exactly. The eigenmodes are computed independently for each axial wavenumber $k_{y}$ using an Arnoldi Krylov technique on the snapshots of the perturbation flow computed during a time marching of (14) (see Donnadieu et al. ${ }^{13}$ for details).

\section{Three-dimensional unstable modes}

Since the base state is symmetric versus $x \rightarrow-x$,

$$
\begin{aligned}
& {\left[u_{B}, 0, w_{B}, \rho_{B}\right](x, z)=\left[-u_{B}, 0, w_{B}, \rho_{B}\right](-x, z),} \\
& {\left[0, \omega_{B}, 0\right](x, z)=\left[0,-\omega_{B}, 0\right](-x, z),}
\end{aligned}
$$

the eigenmodes can be decomposed in a symmetric family (same symmetry as the base state),

$$
\begin{aligned}
& {\left[\tilde{u}_{x}, \tilde{u}_{y}, \tilde{u}_{z}, \tilde{\rho}\right](x, z)=\left[-\tilde{u}_{x}, \tilde{u}_{y}, \tilde{u}_{z}, \tilde{\rho}\right](-x, z),} \\
& {\left[\tilde{\omega}_{x}, \tilde{\omega}_{y}, \tilde{\omega}_{z}\right](x, z)=\left[\tilde{\omega}_{x},-\tilde{\omega}_{y},-\tilde{\omega}_{z}\right](-x, z),}
\end{aligned}
$$

and an antisymmetric family (opposite symmetry to the base state),

$$
\begin{aligned}
& {\left[\tilde{u}_{x}, \tilde{u}_{y}, \tilde{u}_{z}, \tilde{\rho}\right](x, z)=\left[\tilde{u}_{x},-\tilde{u}_{y},-\tilde{u}_{z},-\tilde{\rho}\right](-x, z),} \\
& {\left[\tilde{\omega}_{x}, \tilde{\omega}_{y}, \tilde{\omega}_{z}\right](x, z)=\left[-\tilde{\omega}_{x}, \tilde{\omega}_{y}, \tilde{\omega}_{z}\right](-x, z) .}
\end{aligned}
$$

Symmetric Fig. 5 and antisymmetric Fig. 6 eigenmodes were calculated separately, the symmetries being imposed at each time step during the time marching.

Figs. 5(a), 5(c), 6(a), and 6(c) show the real part of the growth rate $\sigma_{r}^{*}$ scaled by $2 \pi b_{0}^{2} / \Gamma_{0}$, the initial strain imposed by one vortex on the other, as function of the dimensional axial wavenumber $k_{y}^{*}=k_{y} / b_{0}$ scaled by the initial vortex core radius $a_{0}$. Figs. 5(b), 5(d), 6(b), and 6(d) present the same data rescaled by $2 \pi b^{* 2} / \Gamma^{*}$, the instantaneous strain imposed by one vortex on the other and $k_{y}^{*}$ scaled by the instantaneous vortex core radius $a^{*}$. Two instants $\mathrm{Fr}^{-1} t=N t^{*}=1$ (Figs. 5(a), 5(b), 6(a), and 6(b)) and $\mathrm{Fr}^{-1} t=N t^{*}=2$ (Figs. 5(c), 5(d), 6(c), and 6(d)) corresponding to base states plotted in Figs. 1 and 2 are presented displaying several instability bands.

\section{Long wave symmetric instability and the Crow instability}

We first consider the symmetric unstable modes at small wavenumbers at time $\mathrm{Fr}^{-1} t=N t^{*}=2$ displayed in Fig. 5(c). Between $k_{y}^{*} a_{0}=0$ and $k_{y}^{*} a_{0}=0.6$, it shows one band of instability for each Froude number with maximum around $k_{y}^{*} a_{0}=0.3$ with values of the growth rates scaled by the initial strain $\sigma_{r}^{*} 2 \pi b_{0}^{2} / \Gamma_{0}$ decreasing when the Froude number increases $\sigma_{r}^{*} 2 \pi b_{0}^{2} / \Gamma_{0}=0.26$ for $F r=10, \sigma_{r}^{*} 2 \pi b_{0}^{2} / \Gamma_{0}=0.54$ for $F r=5$, and $\sigma_{r}^{*} 2 \pi b_{0}^{2} / \Gamma_{0}=0.94$ for $F r=2$. Fig. 5(d) represents the same data but rescaled by the instantaneous parameters of the dipole, the loci of the maximum $k_{y}^{*} a^{*}$ are shifted to smaller values with increasing stratification and correspond to a maximum around $k_{y}^{*} b^{*}=1$, once rescaled by the instantaneous value of the separation distance between the vortices. 


\section{SYMMETRIC}
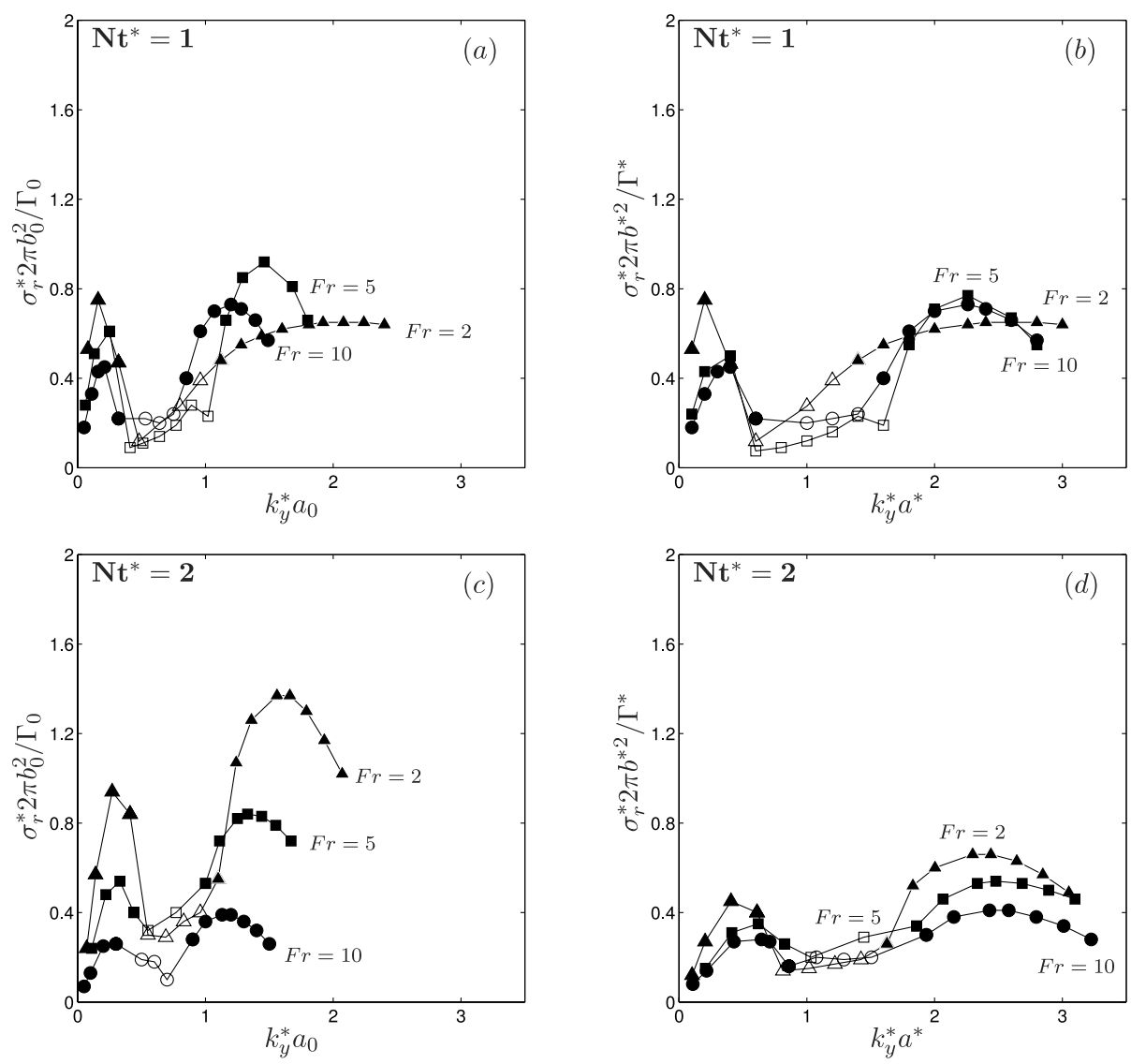

FIG. 5. Growth rates of symmetric modes-growth rates $\sigma_{r}^{*}$ of symmetric modes as function of the axial wavenumber $k_{y}^{*}$ scaled (a) and (c) by the initial values of the radius $a_{0}$, the separation distance $b_{0}$, and the circulation $\Gamma_{0}$ of the vortices and (b) and (d) by the instantaneous values of the radius $a^{*}$, the separation distance $b^{*}$, and the circulation $\Gamma^{*}$ at two instants (a) and (b) $F r^{-1} t=N t^{*}=1$ and (c) and (d) $F r^{-1} t=N t^{*}=2$ for $\operatorname{Re}_{\Gamma_{0}}=2400$ and three Froude numbers $F r=10$ (o), $F r=5$ ( $\square$ ), and $F r=2(\Delta)$. Close symbols correspond to stationary modes whereas open symbols correspond to oscillatory modes.

The values of the maximum growth rates associated with this long wave instability collapse reasonably well for the three Froude numbers, once rescaled by the instantaneous value of the strain (Fig. 5(d)). These scalings of the long wave instability on the separation distance for the wavelength and the instantaneous strain for the growthrate are in agreement with the prediction for the Crow instability. ${ }^{1}$

Fig. 7 represents the axial vorticity (Fig. 7(a)) and the density (Fig. 7(b)) of the leading eigenmode at the maximum of the instability peak for $\mathrm{Fr}=5 \mathrm{at}_{\mathrm{Fr}}^{-1} t=N t^{*}=2$. The vorticity field of this mode is similar to the Crow instability mode which exists in homogeneous fluids since, superimposed on the base flow, it would induce a symmetric displacement of the vortices with an angle of $45^{\circ}$ with no deformation of their internal structures. The density perturbation is located around the vortices at the location where the base flow density gradient is maximum and it also corresponds to the symmetric displacement of the base flow by this Crow-like instability.

\section{Short wave instability}

Let us first discuss instability properties of the frozen base state at the later time $\left(\mathrm{Fr}^{-1} t=\right.$ $N t^{*}=2$ ). For the antisymmetric modes, Fig. 6(c) displays one dominant band of instability for each Froude number between $k_{y}^{*} a_{0}=0.7$ and $k_{y}^{*} a_{0}=1.93$ with maximum growth rates $\sigma_{r}^{*} 2 \pi b_{0}^{2} / \Gamma_{0}=$ 


\section{ANTISYMMETRIC}
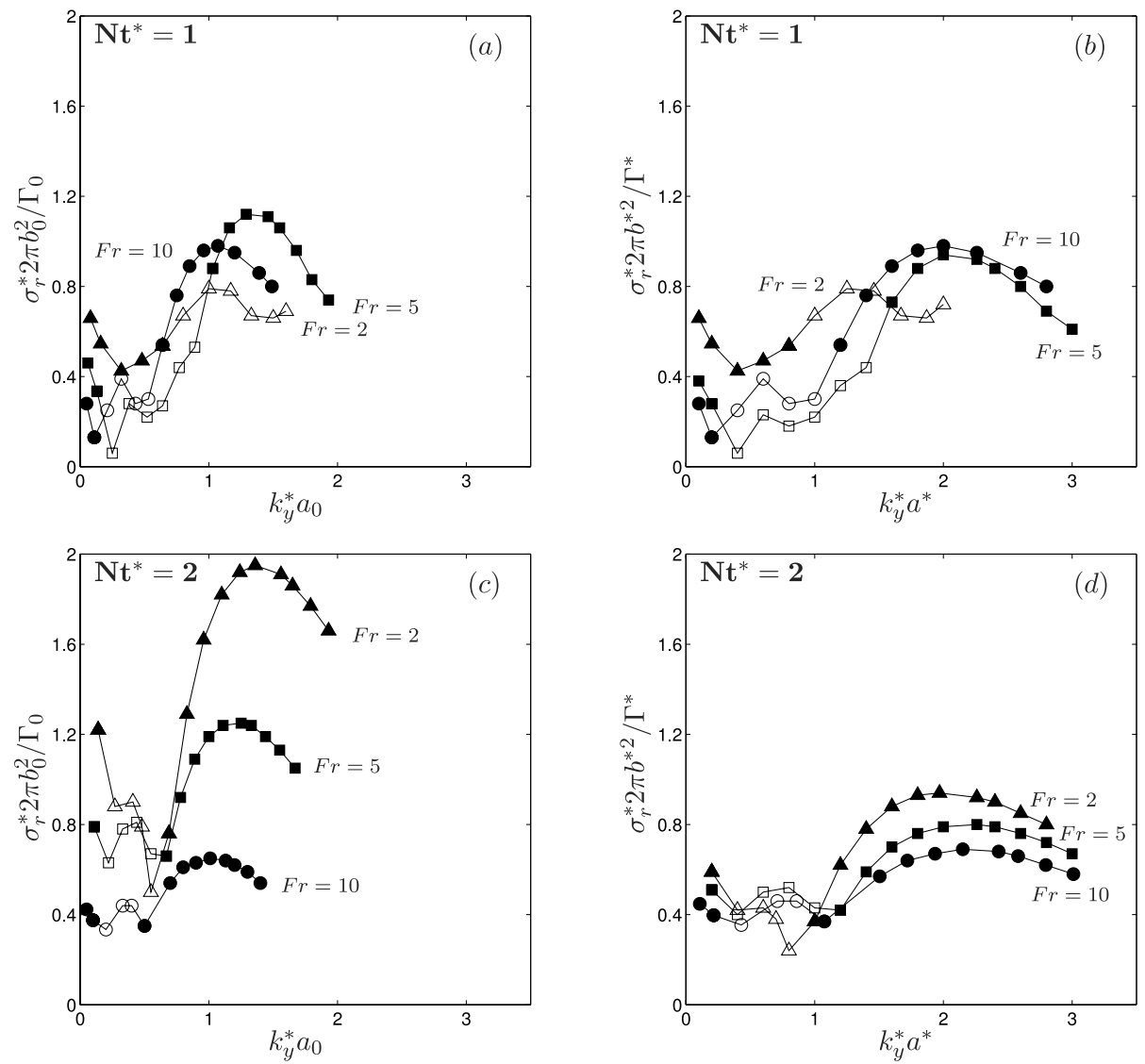

FIG. 6. Growth rates of antisymmetric modes—same legend as Fig. 5 except that the modes are antisymmetric.

$$
\mathrm{Fr}=\mathbf{5} \quad \mathrm{Nt}^{*}=\mathbf{2}
$$
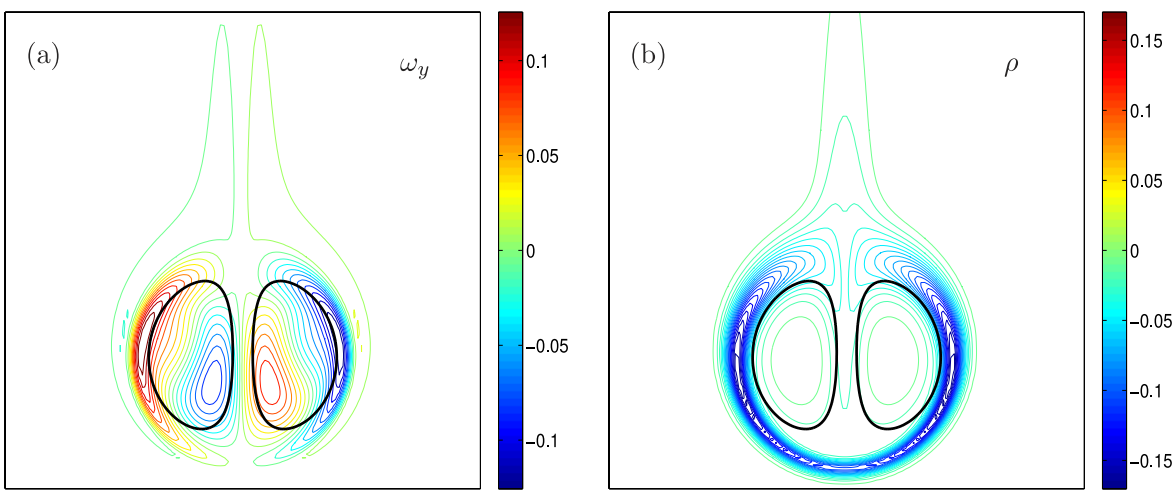

FIG. 7. Long wavelength symmetric instability - Isovalues of the (a) axial vorticity $\omega_{y}$ and (b) density $\rho$ of the eigenmode normed by the square root of energy in the $(x, z)$ plane at the maximum of the unstable peak $k_{y}^{*} a^{*}=0.6$ for $R e_{\Gamma_{0}}=2400$ and $\mathrm{Fr}=5$ at $\mathrm{Fr}^{-1} t=N t^{*}=2$ for the symmetric mode. The heavy black lines correspond to the isocontours $\omega_{B y} / \omega_{B y}^{\max }= \pm \exp (-1)$. The size of the domain shown is $3 b_{0} \times 3 b_{0}$ whereas the computation domain is $12 b_{0} \times 12 b_{0}$. The potential energy represents $67 \%$ of the total energy. 
0.65 at $k_{y}^{*} a_{0}=1$ for $F r=10, \sigma_{r}^{*} 2 \pi b_{0}^{2} / \Gamma_{0}=1.25$ at $k_{y}^{*} a_{0}=1.25$ for $F r=5$, and $\sigma_{r}^{*} 2 \pi b_{0}^{2} / \Gamma_{0}=1.95$ at $k_{y}^{*} a_{0}=1.36$. We observe that the values of the growth rates scaled by the initial value of the strain $\sigma_{r}^{*} 2 \pi b_{0}^{2} / \Gamma_{0}$ at the maximum of the instability bands strongly increase with increasing stratification whereas the $k_{y}^{*} a_{0}$ location of the maximum is slightly shifted to larger values. For the symmetric modes, the trends are similar (Fig. 5(c)) with peaks 3\% smaller than for the antisymmetric modes. These shortwave instability peaks still dominate over the Crow-like instability peaks.

The growth rates of unstable modes as function of the axial wavenumber both rescaled by the instantaneous parameters of the dipole are shown in Figs. 5(d) and 6(d). Once rescaled by the instantaneous values, the curves for three different Froude numbers nearly collapse with, for the antisymmetric mode, a maximum around $k_{y}^{*} a^{*}=2$ with values of $\sigma_{r} 2 \pi b^{2} / \Gamma$ about 1 (and $k_{y}^{*} a^{*}=2.5$ and $\sigma_{r}^{*} 2 \pi b^{* 2} / \Gamma^{*} \simeq 0.5$ for the symmetric mode), which agrees with the prediction of the first resonance branch of the elliptic instability (Tsai and Widnall, ${ }^{3}$ Le Dizès and Laporte ${ }^{19}$ ). The fact that the instability growth rate once rescaled by instantaneous values is slightly weaker when the Froude number is larger is probably due to the viscous dissipation since the larger the Froude number, the longer the time to reach $\mathrm{Fr}^{-1}=N t^{*}=2$ and the stronger the decrease in instantaneous Reynolds number as shown in Fig. 4(c). Similarly, this slight increase of $k_{y}^{*} a^{*}$ where the growth rate is maximum (Fig. 5(d)) may come from the large variation of $a^{*}$ with the Froude number (Fig. 3(a)), it might be more realistic to take the average value of $a^{*}$ and not its final value at $N t^{*}=2$. Fig. 9 displays the axial vorticity and density perturbations of the antisymmetric eigenmode at the maximum of the instability peak $k_{y} a=2.26$ for $F r=5$ at $F r^{-1} t=N t^{*}=2$. The axial vorticity of the eigenmode consists of a dipole in the vortex core and two lobes of opposite sign vorticity at its periphery. This eigenmode corresponds to the deformation of the vortex core in opposite phase to the vortex periphery characteristic of elliptic instability eigenmodes. In Fig. 9(b), there is no density perturbation inside the vortex cores where the density of the base flow is uniform (Fig. 2(d)). The density eigenmode is located at the periphery of the vortex base flow suggesting that it is induced by the transport of the area of strong base flow density gradient at the periphery of the vortices by the perturbation velocity.

At the shorter time $\mathrm{Fr}^{-1} t=N t^{*}=1$, the results are similar to the case $\mathrm{Fr}^{-1} t=N t^{*}=2$ except that, even when initial parameters of the dipole are used for the scaling, the peak of the curves representing the growth rates of the instability as function of the axial wavenumber has similar intensity for the three Froude numbers (Figs. 5(a) and 6(a)).

The axial vorticity of the eigenmode at $\mathrm{Fr}^{-1} t=N t^{*}=1$ for $\mathrm{Fr}=5$ at the maximum of the peak $k_{y} a=2$ is displayed in Fig. 8(a) and is characteristic of the elliptic instability with opposite perturbations between the core and the periphery of the vortex. Eigenmodes of Fig. 8 are similar to the $N t^{*}=2$ case (Fig. 9(a)) except that the size of the perturbation is smaller since the radius of the vortices has grown by viscous diffusion between $\mathrm{Fr}^{-1} t=N t^{*}=1$ and $\mathrm{Fr}^{-1} t=N t^{*}=2$ and

$$
\mathrm{Fr}=5 \quad \mathrm{Nt}^{*}=1
$$
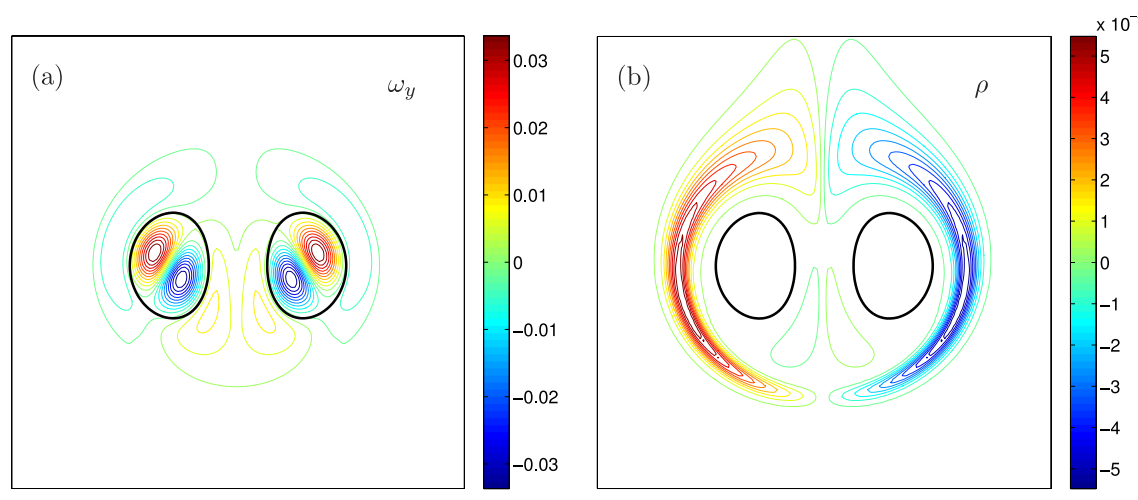

FIG. 8. Short wavelength antisymmetric instability-same as Fig. 7 except that the eigenmode is antisymmetric, $k_{y}^{*} a^{*}=2$, $\mathrm{Fr}^{-1} t=N t^{*}=1$ and the size of the computation domain is $6 b_{0} \times 6 b_{0}$. The potential energy represents $10 \%$ of the total energy. 
$\mathrm{Fr}=5 \quad \mathrm{Nt}^{*}=\mathbf{2}$
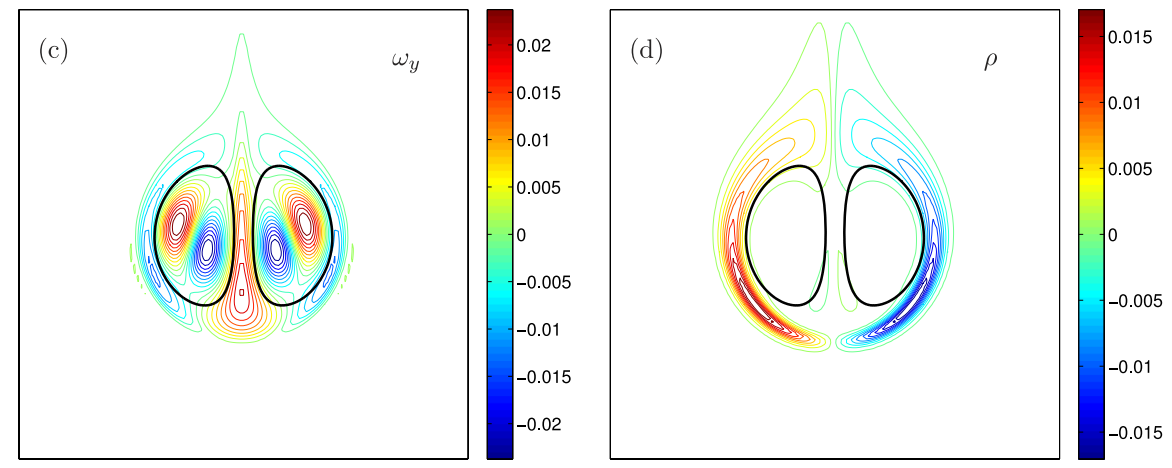

FIG. 9. Short wavelength antisymmetric instability—same legend as Fig. 7 except that the eigenmode is antisymmetric, $k_{y}^{*} a^{*}=2.26$ and the size of the computation domain is $6 b_{0} \times 6 b_{0}$. The potential energy represents $48 \%$ of the total energy.

the perturbation located on each vortex core is more separated since, at that time, the distance $b$ between the vortices is larger (Fig. 1(c) compared to Fig. 2(c)).

For each instant and each Froude number, the leading instability is therefore similar to the elliptic instability of the instantaneous vorticity fields with little direct effect of the stratification. This confirms the interpretation of Nomura et al. ${ }^{12}$ in their direct numerical simulations that, for weak and moderate stratification, the instability mechanism is the elliptic instability. The growth rates scale with the instantaneous value of the strain $\Gamma / 2 \pi b^{2}$ and the wavelengths scale with the instantaneous value the vortex radius $a$, as predicted by the elliptic theory applied on the instantaneous base flow. The growth rates scaled by the initial strain are larger with stronger stratification, which corroborates the results of Nomura et al. ${ }^{12}$ that the main effect of the stratification is to enhance the elliptic instability since the strain is increased by the reduction of the separation distance between the vortices of the base flow. The most unstable eigenmode is the antisymmetric eigenmode and the difference between the growth rates of symmetric and antisymmetric eigenmode increases with $\mathrm{Fr}^{-1} t=N t^{*}$ in agreement with the properties that the antisymmetric elliptic mode leads over the symmetric mode when $a / b$ decreases. ${ }^{20}$

\section{Oscillatory instabilities}

At the instant $\mathrm{Fr}^{-1} t=N t^{*}=2$, the points between $k_{y}^{*} a_{0}=0.2$ and $k_{y}^{*} a_{0}=0.5$ in Fig. 6(c) correspond to an oscillatory instability with a complex growth rate. This instability exists for the three Froude numbers considered. The three curves corresponding to the growth rates as function of the axial wavenumbers rescaled by the instantaneous value of the dipole parameters collapse (Fig. 6(d)), meaning that the growth rate scales with the instantaneous value of the strain. This oscillatory instability also exists for the symmetric modes but it is superseded at small wavenumbers by the Crow instability Fig. 5(c).

Fig. 10(a) represents the axial vorticity of the oscillatory symmetric eigenmode at $k_{y}^{*} a^{*}=1.4$ for $\mathrm{Fr}=5, \mathrm{Fr}^{-1} t=N t^{*}=2$. It consists of a central maximum inside the core of each vortex and two lobes of opposite sign vorticity at the periphery. This mode is reminiscent of an elliptic instability involving Kelvin waves of azimuthal wavenumbers $m=0$ and $|m|=2$ traveling on each vortex described theoretically by Sipp and Jacquin ${ }^{20}$ and observed numerically by Donnadieu et al. ${ }^{13}$

\section{OPTIMAL PERTURBATIONS IN STRATIFIED FLUID}

In the case of strong stratification, i.e., for small Froude numbers, the unsteadiness of the flow questions the validity of the quasi-steady approximation used in Sec. III. In order to study the 


$$
\mathrm{Fr}=5 \quad \mathrm{Nt}^{*}=\mathbf{2}
$$
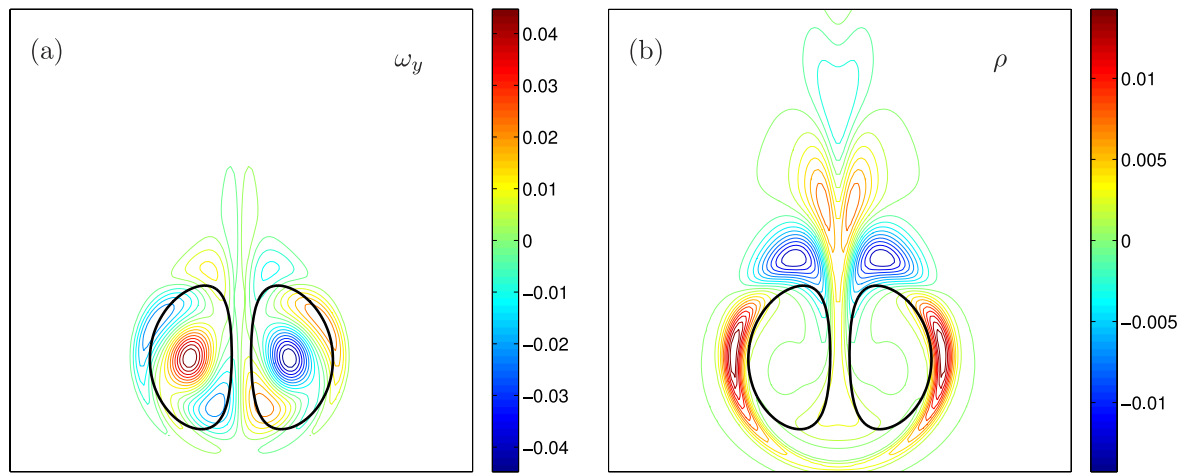

FIG. 10. Oscillatory symmetric instability—same legend as Fig. 7 except that $k_{y}^{*} a^{*}=1.4$. The potential energy represents $40 \%$ of the total energy. Only the real part of the eigenmode is presented. The imaginary part being similar but with perturbation in each vortex turned by $90^{\circ}$, indicating a spiral wave propagating along the vortex axis.

dynamics of this unsteady flow, we explore here a different approach valid with no restriction, in particular when base flow and perturbations evolve on a similar time scale. We compute for each time horizon $\tau$, the initial perturbation that will exhibit the largest gain $G(\tau)$ in total energy by time $\tau$

$$
G(\tau)=\frac{E(\tau)}{E(0)} .
$$

The total energy is the sum of the kinetic energy $E_{c}(\tau)$ and the potential energy $E_{p}(\tau)$

$$
E(\tau)=E_{c}(\tau)+E p(\tau)=\int_{0}^{L_{x}} \int_{0}^{L_{z}}\left(\overline{\mathbf{u}}^{T} \cdot \mathbf{u}+F r^{-2} \bar{\rho} \cdot \rho\right) d x d z
$$

where the $:$ and ${ }^{T}$ superscripts denote, respectively, the complex conjugate and the transposition and $L_{x}$ and $L_{z}$ correspond to the integration domain size in the $x$ and $z$ direction defined in Sec. II A. A direct-adjoint technique (Luchini, ${ }^{21}$ Corbett and Bottaro ${ }^{22}$ ) has been developed in order to compute the optimal perturbations while taking into account the evolution of the base flow. This method requires the computation of both the direct and the adjoint evolution operators obtained by linearizing the Navier-Stokes equation around a time evolving base flow. The scalar product used to construct the adjoint is

$$
\left[\mathbf{f}^{\prime} \mid \mathbf{f}\right]=\int_{0}^{\tau} \int_{0}^{L_{x}} \int_{0}^{L_{z}} \overline{\mathbf{f}}^{\prime T} \cdot \mathbf{f} d x d z d t=\int_{0}^{\tau} \int_{0}^{L_{x}} \int_{0}^{L_{z}}\left(\overline{\mathbf{u}}^{\prime T} \cdot \mathbf{u}+\bar{p}^{\prime} p+\bar{\rho}^{\prime} \rho\right) d x d z d t
$$

where $\mathbf{f}^{\prime}=\left(\mathbf{u}^{\prime}, p^{\prime}, \rho^{\prime}\right)^{T}$ and $\mathbf{f}=(\mathbf{u}, p, \rho)^{T}$ are two complex state vectors. The adjoint of the linearized Navier-Stokes equations is deduced from Equation (11) using the Lagrange identity ${ }^{23,24}$ and rewritten as

$$
\left\{\begin{array}{l}
-\frac{\partial \mathbf{u}^{+}}{\partial t}=\mathbf{\Omega}_{B} \times \mathbf{u}^{+}-\nabla \times\left(\mathbf{U}_{B} \times \mathbf{u}^{+}\right)-\nabla p^{+}-\nabla \rho_{B} \cdot \rho^{+}+\rho^{+} \mathbf{e}_{\mathbf{z}}+R e^{-1} \Delta \mathbf{u}^{+} \\
\nabla \cdot \mathbf{u}^{+}=0, \\
-\frac{\partial \rho^{+}}{\partial t}=\mathbf{U}_{B} \cdot \nabla \rho^{+}-F r^{-2} u_{z}^{+}+R e^{-1} S c^{-1} \Delta \rho^{+},
\end{array}\right.
$$

where $\left[\mathbf{u}^{+}, p^{+}, \rho^{+}\right](x, y, z, t)$ are the adjoint velocity, pressure, and density perturbations and $\left[\mathbf{U}_{B}, \mathbf{\Omega}_{B}\right.$, $\left.\rho_{B}\right]$ are the base flow, velocity, vorticity, and density at time $t$. 


\section{A. Equations and numerical method}

We consider the reduced state vector, $\mathbf{g}=(\mathbf{u}, \rho)^{T}$. In the following, we introduce the inner products $\langle$.$| . \rangle$ of two arbitrary state vectors $\mathbf{g}_{\mathbf{1}}$ and $\mathbf{g}_{2}$ :

$$
\left\langle\mathbf{g}_{1} \mid \mathbf{g}_{2}\right\rangle=\int_{0}^{L_{x}} \int_{0}^{L_{z}} \mathbf{g}_{\mathbf{1}}^{T} \cdot \mathbf{g}_{\mathbf{2}} d x d z
$$

Energy (19) is then defined by

$$
E(t)=\langle\mathbf{g} \mid Q \mathbf{g}\rangle, \quad \text { where } Q=\left(\begin{array}{cccc}
1 & 0 & 0 & 0 \\
0 & 1 & 0 & 0 \\
0 & 0 & 1 & 0 \\
0 & 0 & 0 & \mathrm{Fr}^{-2}
\end{array}\right) .
$$

For a particular time horizon $\tau$, we then look for the initial condition, $\mathbf{g}(0)$, that maximizes gain (18),

$$
G_{\max }(\tau)=\max _{\mathbf{g}(0)}\left(\frac{\langle\mathbf{g}(\tau) \mid Q \mathbf{g}(\tau)\rangle}{\langle\mathbf{g}(0) \mid Q \mathbf{g}(0)\rangle}\right) .
$$

The evolution of any initial condition, $\mathbf{g}(0)$, is obtained by the integration of Navier-Stokes equations linearized around time evolving base flow (5) until the time $\tau$ leading to the reduced state vector $\mathbf{g}(\tau)$. The result of this integration allows the formal definition of the propagator $\Phi(\tau)$,

$$
\mathbf{g}(\tau)=\Phi(\tau) \mathbf{g}(0)
$$

The maximal gain is thus defined by

$$
G_{\max }(\tau)=\max _{\mathbf{g}(0)}\left(\frac{\langle\Phi(\tau) \mathbf{g}(0) \mid Q \Phi(\tau) \mathbf{g}(0)\rangle}{\langle\mathbf{g}(0) \mid Q \mathbf{g}(0)\rangle}\right) .
$$

Similarly, the backward integration of the adjoint linearized Navier Stokes equations when the base flow is evolving in time (21) allows to define the adjoint propagator $\Phi^{+}(\tau)$, which is also the adjoint of $\Phi(\tau)$ with respect to inner product (22). ${ }^{22,24}$ Maximal gain (26) becomes

$$
G_{\max }(\tau)=\max _{\mathbf{g}(0)}\left(\frac{\left\langle\Phi^{+}(\tau) Q \Phi(\tau) \mathbf{g}(0) \mid \mathbf{g}(0)\right\rangle}{\langle\mathbf{g}(0) \mid Q \mathbf{g}(0)\rangle}\right) .
$$

Hermitian matrix $Q(23)$ is non-singular and its inverse is noted $Q^{-1}$, then

$$
\begin{gathered}
G_{\max }(\tau)=\max _{\mathbf{g}(0)}\left(\frac{\left\langle Q Q^{-1} \Phi^{+}(\tau) Q \Phi(\tau) \mathbf{g}(0) \mid \mathbf{g}(0)\right\rangle}{\langle\mathbf{g}(0) \mid Q \mathbf{g}(0)\rangle}\right), \\
G_{\max }(\tau)=\max _{\mathbf{g}(0)}\left(\frac{\left\langle Q^{-1} \Phi^{+}(\tau) Q \Phi(\tau) \mathbf{g}(0) \mid Q \mathbf{g}(0)\right\rangle}{\langle\mathbf{g}(0) \mid Q \mathbf{g}(0)\rangle}\right) .
\end{gathered}
$$

The operator $Q^{-1} \Phi^{+}(\tau) Q \Phi(\tau)$ is hermitian with respect to the inner product, $\langle. \mid Q$.$\rangle ; thus, the$ optimal initial condition is the eigenvector associated to its largest eigenvalue.

The actual procedure to calculate the optimal initial condition and the optimal response at finite times is as follows: to define the evolving base state, the nonlinear two-dimensional Navier-Stokes (Equation (5)) is first integrated till time horizon $t=\tau$, starting from an initial state corresponding to the superposition of two circular Lamb-Oseen vortices of initial aspect ratio $a_{0} / b_{0}=0.2$ as defined previously. The velocity, vorticity, and density gradient fields of the obtained time dependent base flow $\left(\mathbf{U}_{B}, \boldsymbol{\Omega}_{B}, \nabla \rho_{B}\right)$ are stored every $n$ time steps $n \Delta t$ with $\Delta t$ the time step of the computation. The frequency of the base flow storage $n$ is adjusted as a trade-off between memory requirement and precision: the smaller $n$ the larger the precision but the larger the memory used for the storage of the base flow time evolution. The variation of the base flow every time step during the direct and adjoint integrations is regenerated by a linear interpolation between the stored instants. Direct LNS equation (11) is integrated until time horizon $t=\tau$ starting with either divergence-free white noise or an optimal initial condition computed previously for a different axial wavenumber, as initial 
condition $\mathbf{g}(t=0)$, leading to $\Phi(\tau) \mathbf{g}(0)$. Adjoint LNS equation (21) is then integrated backward in time setting $t^{\prime}=\tau-t$ and $\mathbf{g}^{+}=Q \Phi(\tau) \mathbf{g}(0)$ as an initial condition. Adjoint LNS equation (21) is integrated until $t^{\prime}=\tau$ (i.e., back to $t=0$ ). Then, the procedure is reiterated taking as initial condition for the direct integration: $\mathbf{g}(t=0)=Q^{-1} \mathbf{g}^{+}\left(t^{\prime}=\tau\right)$. The successive direct and adjoint integrations are repeated until convergence is obtained ${ }^{22}$ i.e., variations of $\ln (G)$ smaller than $10^{-3}$. This is usually achieved in about 3 to 4 iterations of the forward and backward integration procedure. The optimal energy gains are computed independently for each symmetry. In the following, we define the optimal mean transient growth rate as

$$
\sigma=\ln \left(G_{\max }\right) / 2 \tau
$$

named for short: transient growth rate.

\section{B. Unstratified flows $(F r=\infty)$}

For homogeneous fluids $(F r=\infty)$, the unsteadiness of the base flow is due to viscous diffusion that induces an increase of the vortex core radius $a$. The optimal perturbations have been computed for two distinct time horizons $\tau=4$ and $\tau=10$, both times being larger than $\tau \simeq 2.5$, the typical duration of the transient for a steady dipole determined by Donnadieu et al. ${ }^{13}$ The aspect ratio of the base flow has evolved through viscous diffusion from its initial value $a_{0} / b_{0}=0.2$ to $a / b=0.29$ at $\tau=4$ and $a / b=0.37$ at $\tau=10$.

Fig. 11 displays the dimensional transient growth rate $\sigma^{*}$ scaled either by $\Gamma_{0} / 2 \pi b_{0}^{2}$ the initial strain imposed by one vortex on the other (Figs. 11(a) and 11(b)) or by the instantaneous value of the strain (Figs. 11(c) and 11(d)) as function of the axial wavenumber $k_{y}^{*}$ scaled, respectively, by the initial vortex core radius $a_{0}$ (Figs. 11(a) and 11(b)) and by the instantaneous value of the vortex core radius $a^{*}$ (Figs. 11(c) and 11(d)). The antisymmetric transient growth rates are displayed in Figs. 11(a) and 11(c) and the symmetric ones in Figs. 11(b) and 11(d).

\section{Long wave dynamics}

At low wavenumbers, i.e., for wavenumbers $k_{y}^{*} a_{0}$ roughly smaller than 0.6 , it is striking that the transient growth rates are large for both symmetries whereas in the classical stability analysis, antisymmetric modes are stable for $k_{y}^{*} a_{0}<0.6$ and only symmetric modes exhibit the long-wavelength Crow instability. ${ }^{1}$ Still, the shapes of the transient growth rate curves are different with only the symmetric mode presenting a peak at low wavenumber, reminiscent of the Crow instability.

a. Symmetric perturbations. Fig. 12 displays the optimal initial perturbations and optimal responses at the maximum wavenumber of the low wavenumber peak $k_{y}^{*} a_{0}=0.2$ at $\tau=4$ (Figs. 12(a) and 12(c)) and $\tau=10$ (Figs. 12(b) and 12(d)) for the symmetric case. The axial vorticity being the dominant vorticity component for both optimal initial and final perturbations, the transverse and vertical vorticity components are not reproduced here. The axial vorticity of the optimal response (Figs. 12(c) and 12(d)) consists of a dipole located on the base flow vortices. Superimposed on the base flow, it would induce a symmetric displacement of the vortices along lines inclined at an angle of about $45^{\circ}$ with nearly no deformations of the internal structure of the vortices. This optimal response is therefore similar to the eigenmode of the Crow instability. The size of the region where the vorticity of the optimal response is large has increased between Fig. 12(c) for $\tau=4$ and Fig. 12(d) for $\tau=10$; by doing so, the optimal perturbation keeps corresponding to a $45^{\circ}$ displacement of the cores that grow by diffusion. On the contrary, the optimal initial perturbation is nearly independent of the optimization time horizon $\tau$ and localized on the contracting manifold of the lower stagnation point for both time horizons, i.e., in between the two vortices (Figs. 12(a) and 12(b)). These optimal perturbations can be compared to the investigations of Brion et al. ${ }^{25}$ on the optimal amplification of the Crow instability which develops on a dipole of aspect ratio $a / b=0.2$. However, in their case, the base flow is frozen, the initial perturbation they consider is the adjoint eigenmode which is the optimal perturbation only for time going to infinity. Still, the adjoint Crow mode they have computed is remarkably similar to the present initial optimal perturbations (Figs. 12(a) and 12(b)) with sheets of opposite sign axial vorticity located between 


\section{ANTISYMMETRIC}
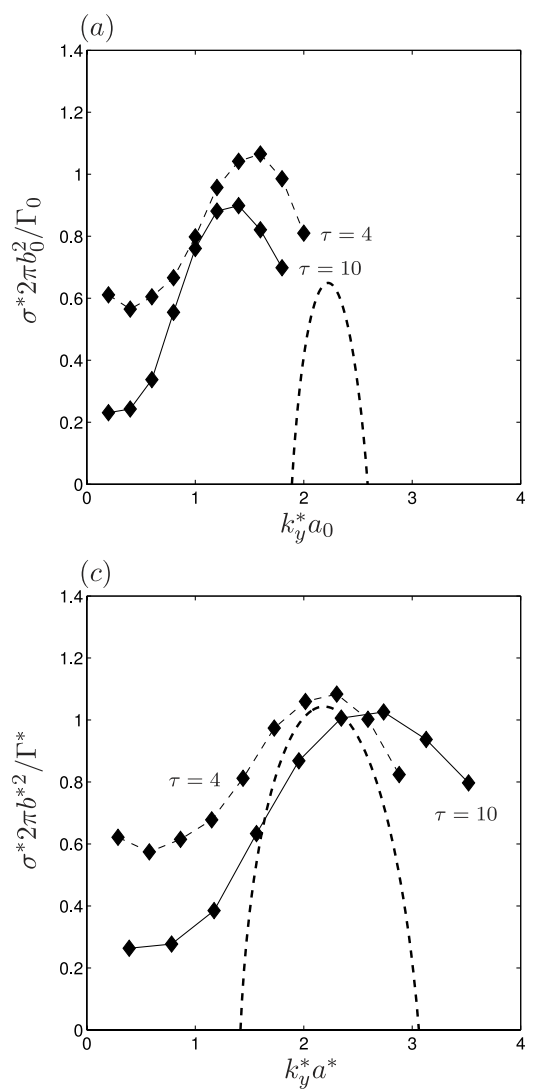

SYMMETRIC
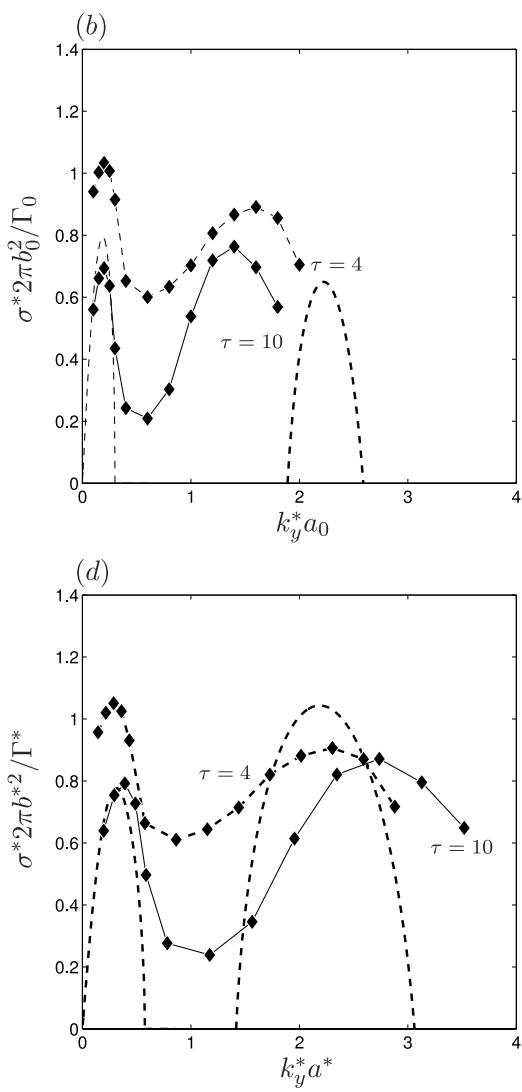

FIG. 11. Optimal mean transient growth rates for $F r=\infty$-transient growth rate $\sigma^{*}$ of the (a) and (c) antisymmetric and (b) and (d) symmetric unstable modes as function of the axial wavenumber $k_{y}^{*}$ scaled by (a) and (b) the initial values of the radius $a_{0}$, the separation distance $b_{0}$, and the circulation $\Gamma_{0}$ of the vortices and the (c) and (d) instantaneous values of the radius $a^{*}$, the separation distance $b^{*}$, and the circulation $\Gamma^{*}$ at time horizons $\tau=4(----)$ and $\tau=10(-\downarrow)$. Thin dashed lines correspond to the theoretical prediction of Crow for a pair of vortex filaments for an inviscid and homogeneous fluid and thick dashed lines are the inviscid theory of Le Dizès and Laporte ${ }^{19}$ for a pair of Lamb-Oseen vortices in a homogeneous fluid with the viscous correction proposed by Donnadieu et $a l .{ }^{13}$ computed for $\operatorname{Re}_{\Gamma}=2400$ for $a / b=0.2$. For (a) and (b), these theoretical predictions are computed with initial parameters of the dipole and for (c) and (d) with instantaneous parameters of the dipole at $\tau=4$ and $\tau=10$.

the vortices. Also their final perturbation is the Crow instability mode similar to the present optimal final perturbation but more concentrated since the base flow diffusion is not taken into account ${ }^{25}$ in their study. Such opposite sign vorticity sheets in the axial vorticity correspond to a streamwise jet between the two base flow vortices. At low wavenumber, this streamwise jet induces spanwise vorticity perturbations displacing the vortices in a way consistent with the Crow instability.

The predicted growth rate of the Crow instability for a pair of vortex filaments for an inviscid and unstratified flow is plotted with dashed lines in Figs. 11(b) and 11(d). It predicts a maximum growth rate at $k_{y}^{*} a_{0}=0.19$, corresponding to $k_{y}^{*} b_{0}=1.05$. This theoretical prediction is in good agreement both in location in $k_{y}^{*} a_{0}$ and in height with the transient growth rate computed at both time horizons, with a better agreement at $\tau=10$ where the transient growth rate has decreased by $38 \%$ compared to $\tau=4$.

b. Antisymmetric perturbations. Donnadieu et al. ${ }^{13}$ show that antisymmetric perturbations may exhibit strong transient growth at early time horizons even though they are stable at large time. They find transient growth rate for $k_{y}^{*} a_{0}=0.2$ of 0.6 which is remarkably similar to the mean growth rate obtained at the same time horizon in the present study in Fig. 11 (about half the growth rate of the symmetric perturbation). In their study, the base flow is frozen, and the dipole aspect 
$\tau=4$

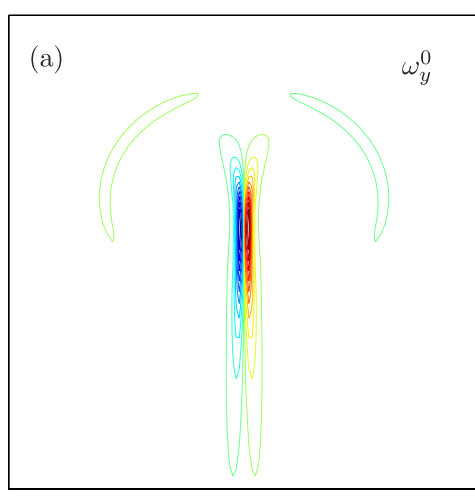

(c)

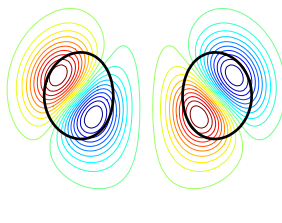

$\tau=10$
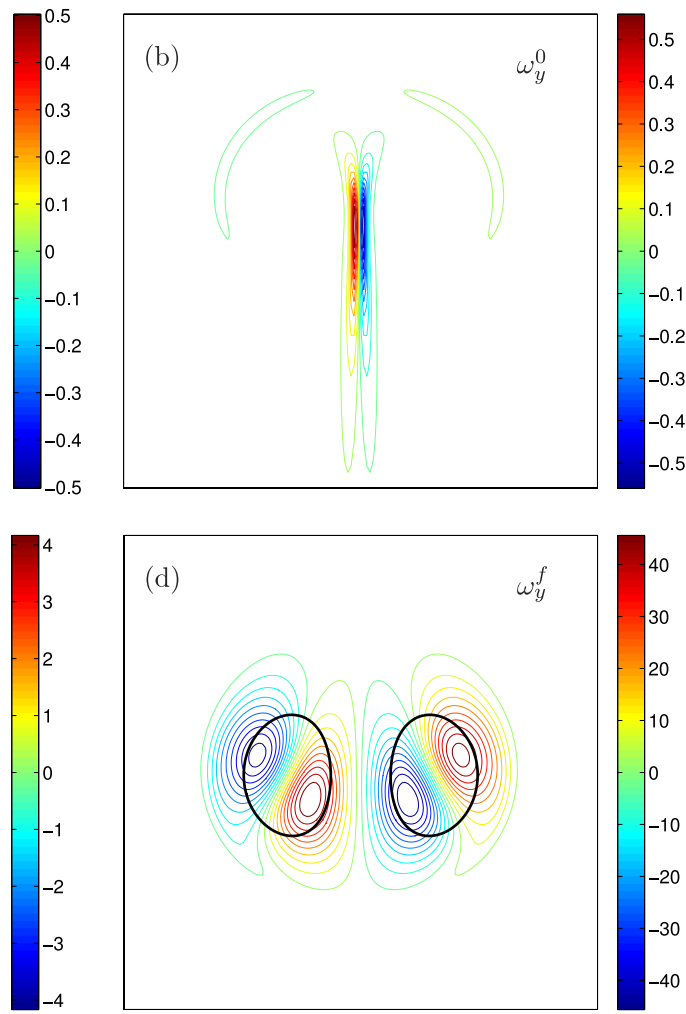

FIG. 12. Symmetric optimal perturbations for $F r=\infty$ at the maximum of the long-wavelength peaks $k_{y}^{*} a_{0}=0.2$-isovalues of the axial vorticity of the (a) and (b) optimal initial perturbation $\omega_{y}^{0}$ and the (c) and (d) optimal response $\omega_{y}^{f}$ at time horizons (a) and (c) $\tau=4$ and (b) and (d) $\tau=10$ in the $(x, z)$ plane normed by the square root of the total energy of the optimal initial perturbation. The heavy black lines correspond to the isocontours $\omega_{B y} / \omega_{B y}^{\max }= \pm \exp (-1)$. The domain shown is $6 b_{0} \times 6 b_{0}$ (whereas the computational domain is $12 b_{0} \times 12 b_{0}$ ) and has been shifted down vertically to accommodate the downwards displacement of the dipole.

ratio is $a / b=0.2$. At larger horizon time, the aspect ratio has evolved to 0.37 through viscous diffusion, and the prediction of Donnadieu et al. ${ }^{13}$ overestimates by a factor 3 at $\tau=10$ the exact transient growth rate. For both time horizons, the transient growth rate presents no peak and is even maximum at $k^{*}=0$ in contrast to the symmetric case. Antisymmetric optimal initial perturbation and final response are not shown for the seek of concision but, as for the symmetric case, the optimal response corresponds to an antisymmetric displacement of the vortices with no deformation of the vortex cores giving a perturbation dominated by spanwise vorticity with a dipolar shape in each vortex. The optimal initial perturbation (not shown) is located on the contracting manifolds of both the upper and the lower stagnation point, and it also differs from the symmetric case since it is dominated by the vertical vorticity (streamwise vorticity) and not by the spanwise vorticity. This antisymmetric perturbation induces a meandering of the vortex dipole along its span associated with a tilt.

\section{Short wave dynamics}

At large wavenumbers, i.e., for $k_{y}^{*} a_{0}>0.6$ for both symmetries and both time horizons, we observe in Fig. 11 a well separated band with an isolated maximum, and we will show in the following that this corresponds to the elliptic instability. For both symmetries, the wavenumber at the maximum $k_{y}^{*} a_{0}=1.6$ at $\tau=4$ is shifted to the smaller value $k_{y}^{*} a_{0}=1.4$ at larger time horizon $\tau=10$. The width of the peak narrows when time horizon increases from $\tau=4$ to $\tau=10$. This narrowing with time suggests that the strong selectivity of the elliptic instability appears progressively 
when the time horizon gets large. The maximum of the transient growth rates of the antisymmetric perturbation is larger than that of the symmetric mode with a difference of $20 \%$ at $\tau=4$ and of $15 \%$ at $\tau=10$ at the maximum of the bands. We confirm the experimental work of Leweke and Williamson ${ }^{27}$ who observed at $t \Gamma / 2 \pi b^{2}=7.5$ that the leading eigenmode is antisymmetric.

Fig. 13 displays the optimal initial perturbations and optimal responses near the maximum of the peaks $k_{y}^{*} a_{0}=1.2$ at $\tau=4$ (Figs. 13(a) and 13(c)) and $\tau=10$ (Figs. 13(b) and 13(d)) for the antisymmetric case. As for the long wave dynamics, only the dominant axial vorticity component is represented. The symmetric perturbations, less unstable than, but similar to, the antisymmetric ones, are not displayed for the sake of brevity.

At the two instants, the optimal response is located inside the cores of the vortices with an axial vorticity perturbation which consists of a dipole nested inside the core and surrounded by two lobes of opposite vorticity at the periphery of each base flow vortex (Figs. 13(c) and 13(d)). Superimposed on the base flow, this optimal response would induce a displacement of the inner and outer parts of each vortex core in opposite directions. The optimal response inside each vortex core is similar to the eigenmode of the first elliptic instability resonance discussed in Fig. 8(a). At $\tau=10$, the optimal response (Fig. 13(d)) is spatially more extended than at $\tau=4$ but comparison with the bold line visualizing the actual instantaneous size of the cores at each time horizon shows that the optimal response is quasi-statically adjusted to the vortex core size at the final time, core that has grown by viscous diffusion. For $\tau=10$, the outer parts of the axial vorticity of the optimal response (Fig. 13(d)) located between the two vortices have merged since, at that time, the base flow vortices are in contact. The optimal initial antisymmetric perturbations (Figs. 13(a) and 13(b)) are

$$
\tau=4
$$
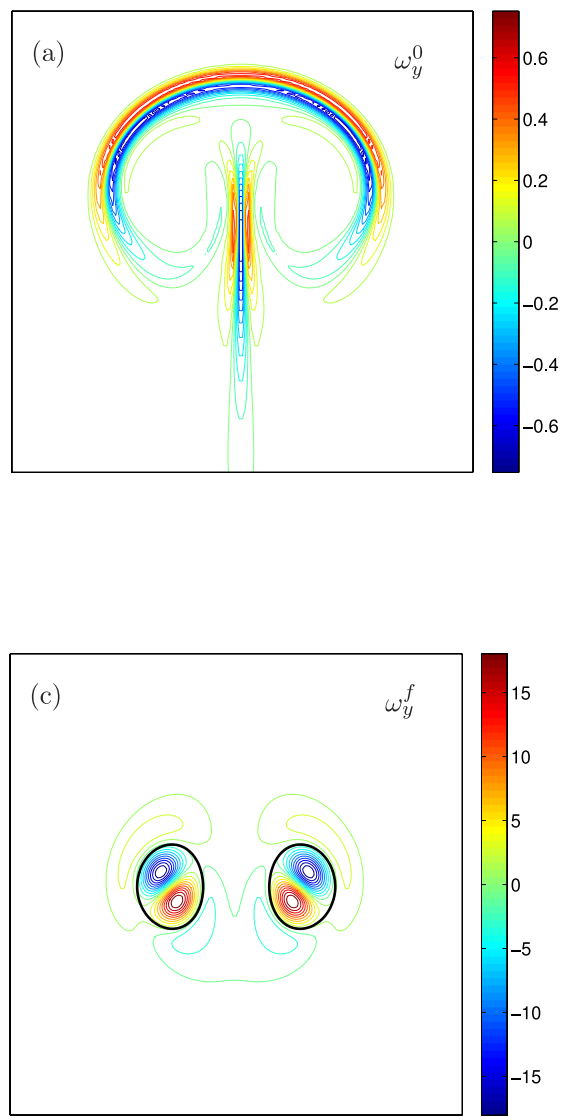

$\tau=10$
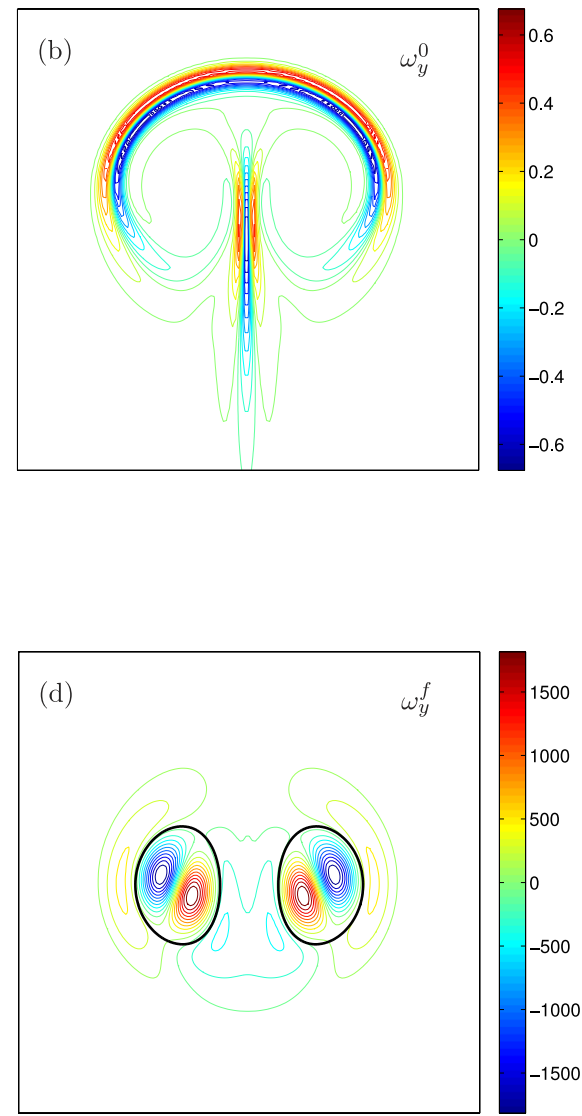

FIG. 13. Antisymmetric optimal perturbations for $F r=\infty$ at the maximum of the short-wavelength peaks-same legend as Fig. 12 except that $k_{y}^{*} a^{*}=2.83$ and the perturbations are antisymmetric. 
nearly identical: for both time horizons $\tau=4$ and $\tau=10$, the axial vorticity is intense on the contracting manifolds of both upper and lower stagnation points (and similar to the low wavenumber antisymmetric optimal initial perturbations that were not shown in Sec. IV B 1).

For the symmetric case, the optimal initial perturbations (not reproduced here) are similar to the long wave ones (Fig. 12): the axial vorticity is intense only on the contracting manifold of the lower stagnation point. It is noticeable that the initial perturbation shape is nearly independent of the time horizon and depends only on the symmetry which activates different stagnation points. The symmetric optimal responses at $\tau=4$ and $\tau=10$ (not shown) are similar to the antisymmetric ones except that the sign of the axial vorticity of the perturbations which develop on one vortex is opposite to the one that develop on the other vortex at the symmetric location.

The inviscid theoretical prediction for the elliptic instability given by Le Dizès and Laporte ${ }^{19}$ for a pair of Lamb-Oseen vortices of aspect ratio $a / b=0.2$ in a homogeneous fluid corrected by the viscous damping derived by Donnadieu et al. ${ }^{13}$ computed for $R e_{\Gamma}=2400$ is plotted in Figs. 11(a) and 11(b) with light grey dashed lines. The maximum of the unstable band predicted by the theory is at the wavenumber $k_{y}^{*} a^{*}=2.26$. This value is larger than the value of the most amplified wavenumber scaled by the initial vortex radius $k_{y}^{*} a_{0}$ computed at $\tau=4$, which is shifted to an even shorter value at the larger horizon time $\tau=10$. Figs. 11(c) and 11(d) display the same transient growthrate $\sigma^{*}$ as function of the axial wavenumber, now rescaled by instantaneous values of the dipole parameters $a^{*}, b^{*}$, and $\Gamma^{*}$. When scaled by instantaneous radius $a^{*}$, the maximum wavenumber realigns with that predicted by the elliptic instability theory ${ }^{13}$ for $\tau=4$ (Fig. 11(c)). The maximum of the peak at $\tau=10$ is at a slightly larger value $k_{y}^{*} a^{*}=2.7$. When the final instantaneous parameters of the dipole are used, not only do the peaks of the shortwave length mode realign but heights also correspond to the theoretical prediction.

This agreement shows two important facts, first that for both symmetries, the peak in transient growth rate around $k_{y}^{*} a^{*}=2.4$ corresponds to the elliptic mode and that it is quasi-statically lead by instantaneous values of the dipole parameters. In reality, the transient growth rate takes into account the entire history; hence, one may imagine that averaged values of $\bar{a}, \bar{b}$, and $\bar{\Gamma}$ might yield a better collapse of the data. We find that the agreement is slightly improved but not significantly and not worth the complexity (not shown).

The theoretical prediction of Le Dizès and Laporte ${ }^{19}$ is valid in the limit $a / b \rightarrow 0$ and in particular does not include the perturbation coupling from one vortex to the other and as a result, symmetric and antisymmetric modes have the same growth rate at this level of approximation.

To better compare the optimal perturbation and the elliptic instability, a numerical linear stability analysis of a dipole of initial aspect ratio $a_{0} / b_{0}=0.2$ frozen at $\tau=10$ has been performed for $R e_{\Gamma}=2400$. Fig. 14 compares the transient growth for the antisymmetric perturbation at $\tau=10$ with the quasi-steady linear growth rate computed in Sec. III. Both computations agree remarkably well, and the main difference is a slight shift toward longer wavelength of the maximum transient growth. The axial vorticity of the eigenmode $\omega_{y}$ associated with the maximum instantaneous growth rate at $\tau=10$ is represented in Fig. 14(b) and is remarkably similar to the optimal response at $k_{y}^{*} a_{0}=1.2$ (Fig. 13(d)). This confirms that the linear instability dominates the optimal response and is then due to the elliptic instability of the time varying base flow.

This is consistent with Sipp et al., ${ }^{26}$ who derive theoretically the instantaneous growth rate of a perturbation flow field when the base flow evolves with time. Due to the diffusion of the base flow, the dipole aspect ratio $a / b$ increases with time. Using a shape assumption, the growth rate of the perturbation at time $t$ is then the growth rate of the leading elliptic mode evaluated for a frozen base flow corresponding to the dipole aspect ratio at time $t$ corrected by the change in shape of the corresponding eigenmode with respect to $a / b$. For $R e_{\Gamma}=2750$, Leweke and Williamson ${ }^{27}$ observed an antisymmetric elliptic mode at $k_{y}^{*} b^{*}=8.16$ between $t^{*} \Gamma^{*} / 2 \pi b^{* 2}=4.9$ and $t^{*} \Gamma^{*} / 2 \pi b^{* 2}=7.5$; the observed amplification rate is $\sigma^{*} 2 \pi b^{* 2} / \Gamma^{*}=0.94$. Sipp and Jacquin ${ }^{20}$ compute the growth rate of the perturbation characterized by $k_{y}^{*} b^{*}=8.16$ which is found to be $\sigma^{*} 2 \pi b^{* 2} / \Gamma^{*}=0.99$ for $R e_{\Gamma}=2750$. This is consistent with our rigorous optimisation calculation since (Fig. 11(c)) the antisymmetric optimal perturbation at $\tau=4$ corresponds to a mean growth rate of $\sigma^{*} 2 \pi b^{* 2} / \Gamma^{*}=1.06$, the optimal wavenumber being $k_{y}^{*} a^{*}=2.33$ corresponding to $k_{y}^{*} b=8.16$ since $a / b=0.29$ and at $\tau=10$ corresponds to a mean growth rate of $\sigma^{*} 2 \pi b^{* 2} / \Gamma^{*}=1.0$, the optimal wavenumber being 

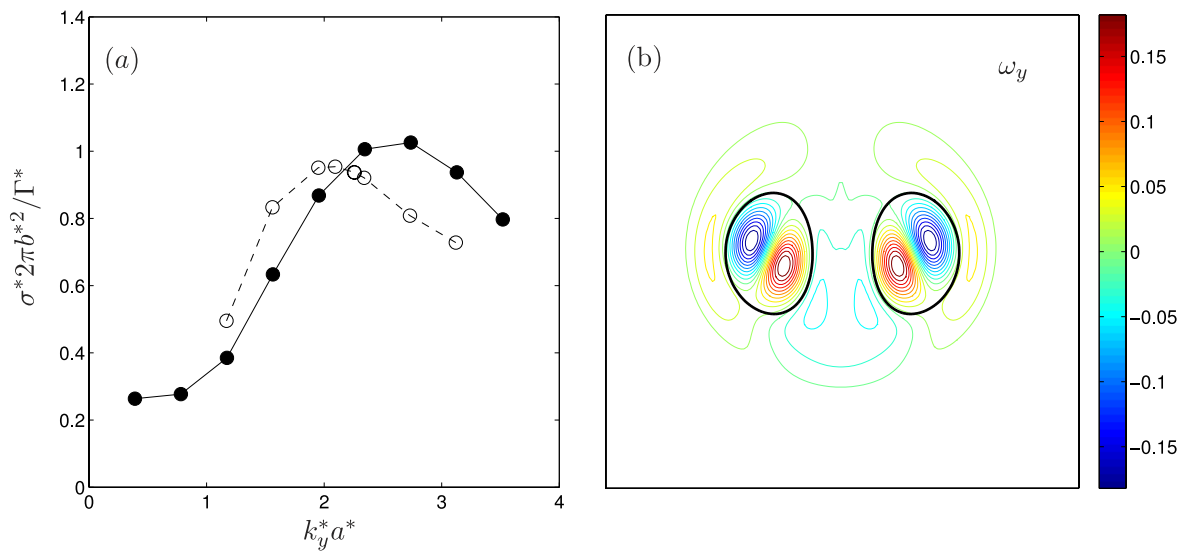

FIG. 14. Comparison between linear stability of the frozen state at $\tau=10$ and optimal transient growth at $\tau=10$ for homogeneous flows-(a) optimal mean transient growth rates at $\tau=10(-\bullet-)$ and growth rates computed by a linear stability analysis with a 2D base flow frozen at $\tau=10(--\circ--) \sigma^{*}$ of antisymmetric perturbations as function of the axial wavenumber $k_{y}^{*}$, both scaled by instantaneous parameters of the dipole for $\operatorname{Re}_{\Gamma_{0}}=2400$ and $F r=\infty$. (b) Isovalues of the axial vorticity $\omega_{y}$ of the eigenmode in the $(x, z)$ plane at the maximum of the unstable peak of the linear stability analysis at $\tau=10 k_{y}^{*} a^{*}=2.83$ (Fig. 14(a)).

$k_{y}^{*} a^{*}=2.83$ corresponding to $k_{y}^{*} b=8.16$ since $a / b=0.37$. Both the value of the growth rate and the evolution of the optimal mean perturbation in the present exact computation legitimate the shape assumption made in Sipp and Jacquin. ${ }^{20}$

\section{Stratified flows}

When the fluid is stably stratified, the unsteadiness of the base flow is due, on top of the viscous diffusion, to buoyancy effect. Fig. 15 displays the optimal mean transient growth rates $\sigma^{*}$ scaled by $2 \pi b_{0}^{2} / \Gamma_{0}$, the initial strain imposed by one vortex on the other, for the antisymmetric (Figs. 15(a) and 15(c)) and symmetric (Figs. 15(b) and 15(d)) cases at two instants $\tau=4$ (Figs. 15(a) and 15(b)) and $\tau=10$ (Figs. 15(c) and 15(d)), for $R e_{\Gamma_{0}}=2400$ and for five Froude numbers $F r=\infty, 10,5$, 2 , and 1. Different stratifications are here compared at the same time nondimensionalized by the dynamical time and not the buoyancy time scale, $\frac{1}{N}$ in order to keep a similar diffusion effect. To compare with the quasi-steady linear stability analysis in Sec. III that were made at fixed time nondimensionalized by buoyancy time scale, Table I gives the value of $N t^{*}=\mathrm{Fr}^{-1} t$ for $\tau=4$ and $\tau=10$ and different Froude numbers. For $F r=\infty$, the unsteadiness is solely due to viscosity, and results have already been shown and discussed in Fig. 11. For $F r=1$, the optimal perturbations have been computed only at $\tau=4$ since, at the time $\tau=10$, the base flow primary vortices have been completely detrained.

For $\tau=4$ and $\tau=10$, it is striking that the transient gain is very similar for all the Froude numbers above $F r=2$ suggesting that stratification does not affect the instability mechanism down to $F r=2$. Even for $F r=2$, stratification increases slightly the growth rate of all the wavenumbers keeping the main trends similar. The effect of density perturbations on the dynamics may be evaluated by comparing the respective contribution of the potential and kinetic energy to the optimal initial perturbation and the optimal response at different instants. Such data are collected in Table III for $k_{y}^{*} a_{0}=0.2$ and in Table IV for $k_{y}^{*} a_{0}=1.6$ of Appendix B.

In all the cases, even at late time $\tau=10$ for $F r=2$ except for Froude number equal to 1 at $\tau=4$ (see Tables III and IV), the potential energy of the initial perturbation is less than $0.5 \%$. The effect of the initial density perturbation is therefore marginal and can be safely ignored for all times studied and all Froude numbers except $F r=1$. For Froude number equals to 1, the potential energy in the initial perturbation is still limited between $5 \%$ and $11 \%$ of the total energy, the highest value being reached by the symmetric mode at small wavenumber. Therefore, even for $F r=1$, the optimal initial perturbation keeps being led by inertial effects and not by gravity effects and for simplicity, 
(a)

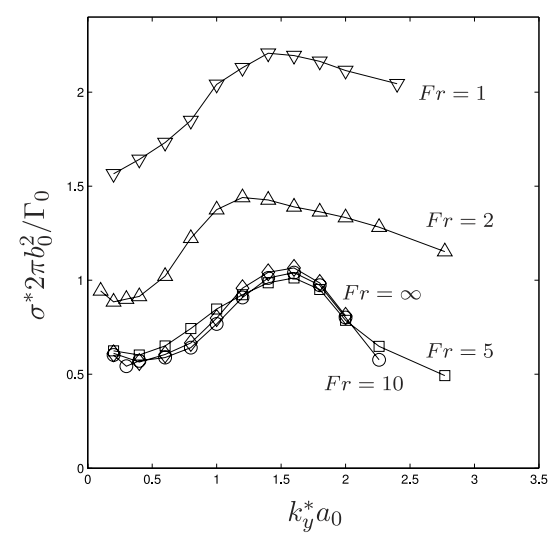

(b)

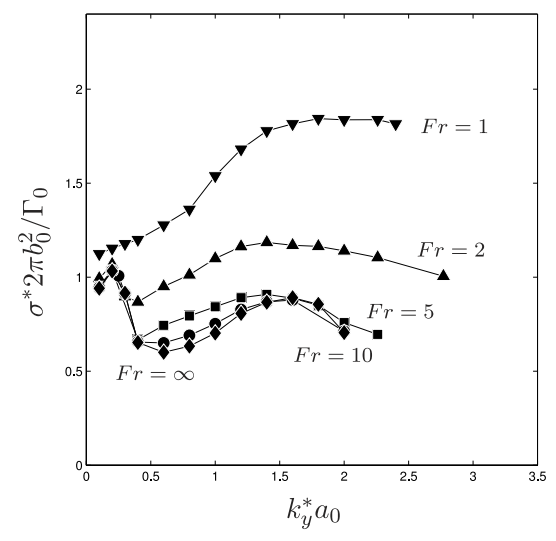

$\tau=10$ (c)

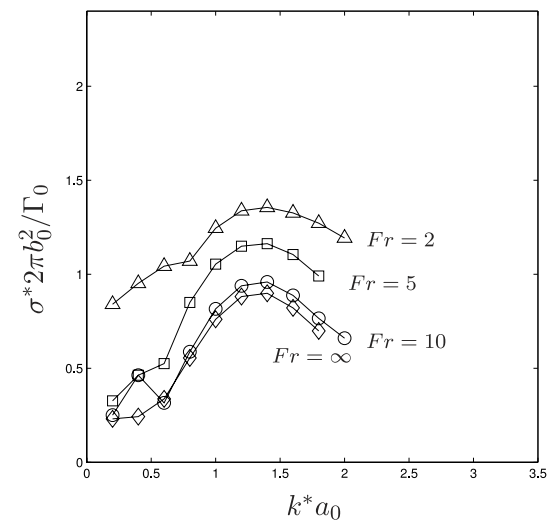

(d)

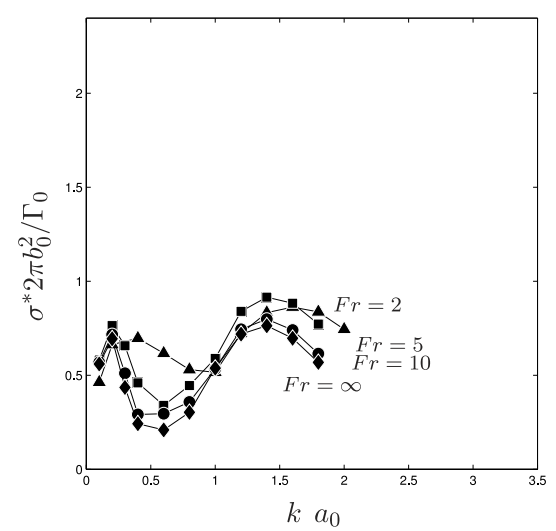

FIG. 15. Optimal mean transient growth rates $-\sigma^{*}$ at two instants (a) and (b) $\tau=4$ and (c) and (d) $\tau=10$ for the (a) and (c) antisymmetric and (b) and (d) symmetric cases as function of the axial wavenumber $k_{y}^{*}$ scaled by the initial values of the radius $a_{0}$, the separation distance $b_{0}$, and the circulation $\Gamma_{0}$ of the vortices for $\operatorname{Re}_{\Gamma_{0}}=2400$ and for $F r=\infty(\diamond, \diamond), F r$ $=10(\circ, \bullet), F r=5(\square, \boldsymbol{\square}), F r=2(\Delta, \mathbf{\Delta})$, and $F r=1(\nabla, \mathbf{v})$.

initial density perturbations field is not presented and discussed here even for $F r=1$. Similarly, the potential energy of the optimal response is negligible for $\mathrm{Fr}>2$ at time horizon $\tau=4$ and $F r>5$ at time horizon $\tau=10$ (Tables III and IV). But it cannot be neglected for both symmetries and both wavenumbers $\left(k_{y}^{*} a_{0}=0.2\right.$ and $\left.k_{y}^{*} a_{0}=1.6\right)$ with $F r=1$ at $\tau=4$ and $F r=5$ and $F r=2$ at $\tau=10$ for which it represents between $30 \%$ and $50 \%$ of the total energy of the optimal response. It cannot be neglected either for the symmetric high wavenumber case at $F r=2$ and $\tau=4$. This result based on potential energy contribution is coherent with the fact that mean growth rate curves (Fig. 15) at time horizon $\tau=4$ depart from the neutral case $F r=\infty$ only for $F r$ smaller than 2 and at time horizon $\tau=10$ only for $F r$ smaller than 5. For the two instants $(\tau=4, \tau=10)$ for which optimal perturbations have been computed, the above discussion shows that potential energy becomes significant in the optimal response only when $N t^{*}$ is larger than 2 , a feature coherent with the quasi-steady linear stability analysis (Sec. III) for which instantaneous eigenmode involves both density and inertial effects only when $N t^{*}$ is larger than 2 . 
TABLE I. Values of $N t^{*}=F r^{-1} t$ for each Froude number at $\tau=4$ and $\tau=10$.

\begin{tabular}{llllr}
\hline \hline$\tau \backslash F r$ & 10 & 5 & 2 & 1 \\
\hline 4 & 0.4 & 0.8 & 2 & 4 \\
10 & 1 & 2 & 5 & 10 \\
\hline \hline
\end{tabular}

\section{Long wavelength dynamics}

At low wavenumbers, as for the unstratified flow, the transient gains are large for both symmetries.

a. Symmetric perturbations. For the symmetric case, the optimal transient growth rate presents a peak around $k_{y}^{*} a_{0}=0.2$ for the Froude numbers larger than or equal to 2 at $\tau=4$ and larger than or equal to 5 at $\tau=10$. This peak is of similar strength for all Froude numbers, and we shall see that, as already discussed for $F r=\infty$, it corresponds to the Crow instability. Figs. 16 and 17 display the axial vorticity of the optimal perturbations (which is presently the largest component of vorticity perturbations) at $\tau=4$ (Fig. 16) and $\tau=10$ (Fig. 17) for the symmetric case at $k_{y}^{*} a_{0}=0.2$ close to the maximum optimal gain for the three Froude numbers $\mathrm{Fr}=5,2$, and 1 .

At the earlier time $\tau=4$ for $F r=2$, the axial vorticity of optimal initial and final perturbations is not affected by stratification and is similar to the unstratified flow $(F r=\infty)$. The axial vorticity

$$
F r=2
$$
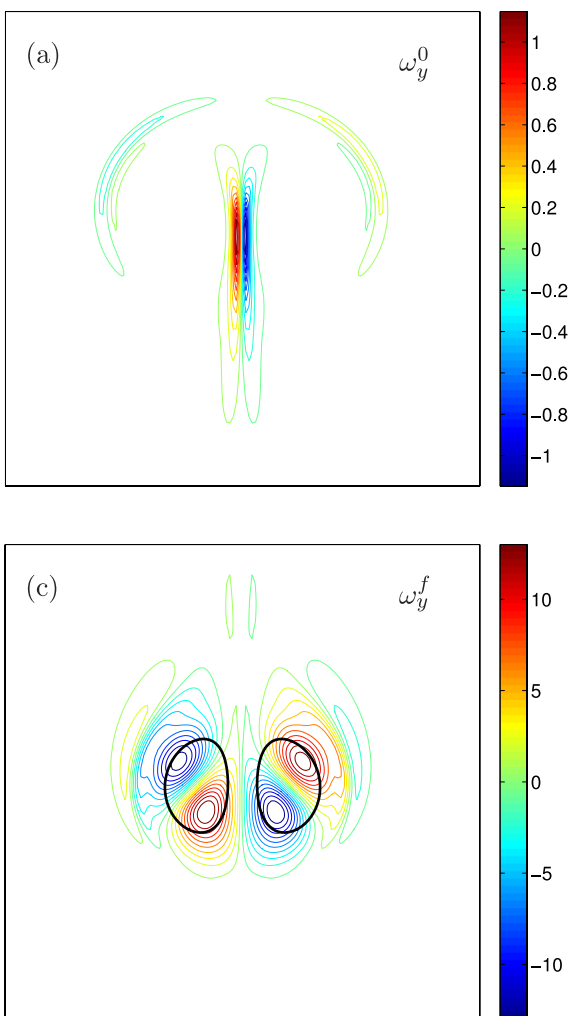

$\mathrm{Fr}=1$
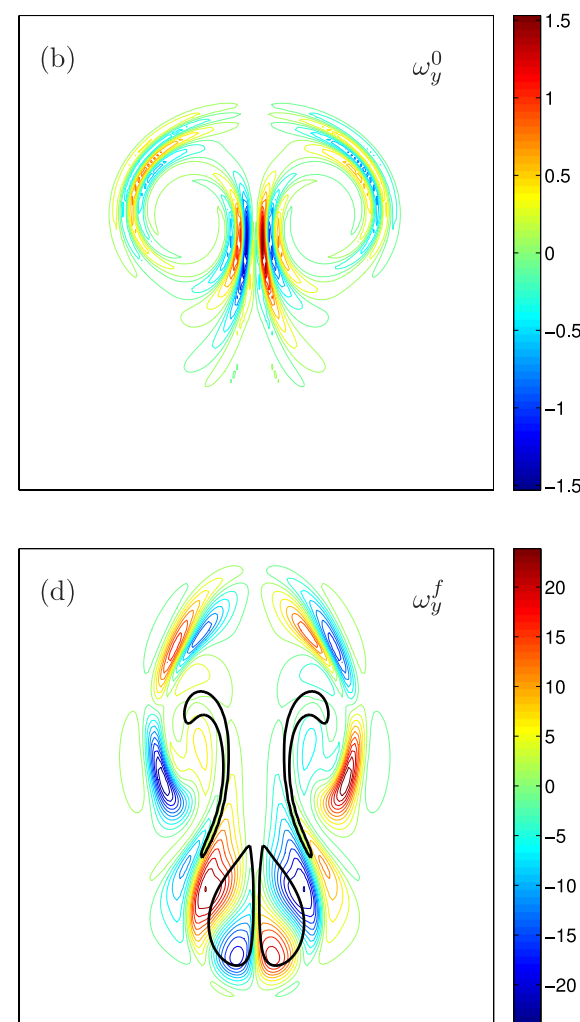

FIG. 16. Transient Crow type instability: symmetric optimal perturbations for the long-wavelength peaks $\left(k_{y}^{*} a_{0}=0.2\right.$ at $\tau=4$ ) -isovalues of the axial vorticity of the (a) and (b) optimal initial perturbations $\omega_{y}^{0}$ and the (c) and (d) optimal responses $\omega_{y}^{f}$ at the time horizon $\tau=4$ in the $(x, z)$ plane normed by the total energy of the optimal initial perturbation. For (a) and (c) $F r=2$ and (b) and (d) $F r=1$. The thick black lines materialize the "core" of the vortices and correspond to the isocontours $\omega_{B y} / \omega_{B y}^{\max }= \pm \exp (-1)$. The size of the domain shown is $3.5 b_{0} \times 3.5 b_{0}$ whereas the computation domain is $12 b_{0} \times 12 b_{0}$ and the domain is shifted to follow the vortices in their descent. 


$$
\tau=10
$$

$$
F r=5
$$
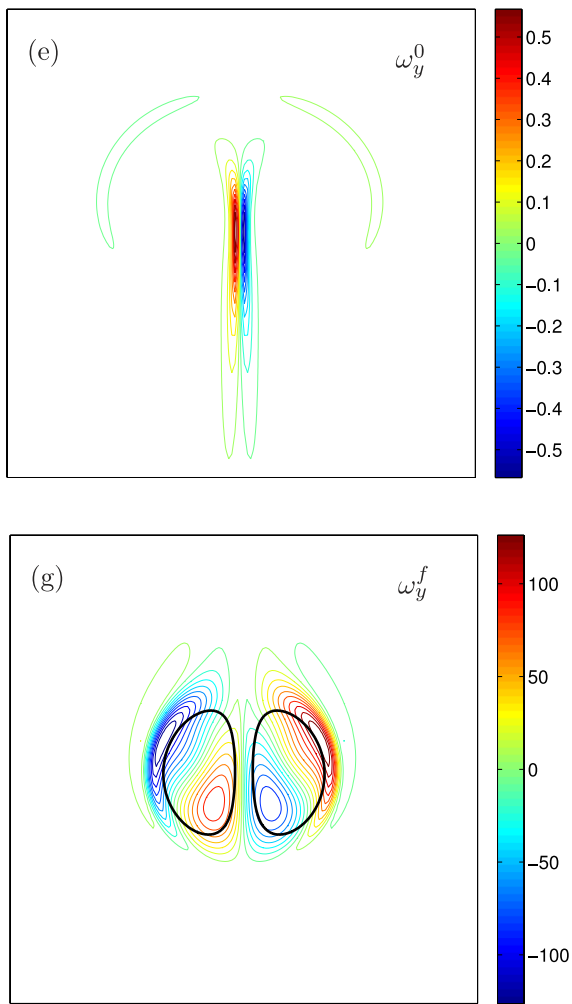

$\mathrm{Fr}=2$
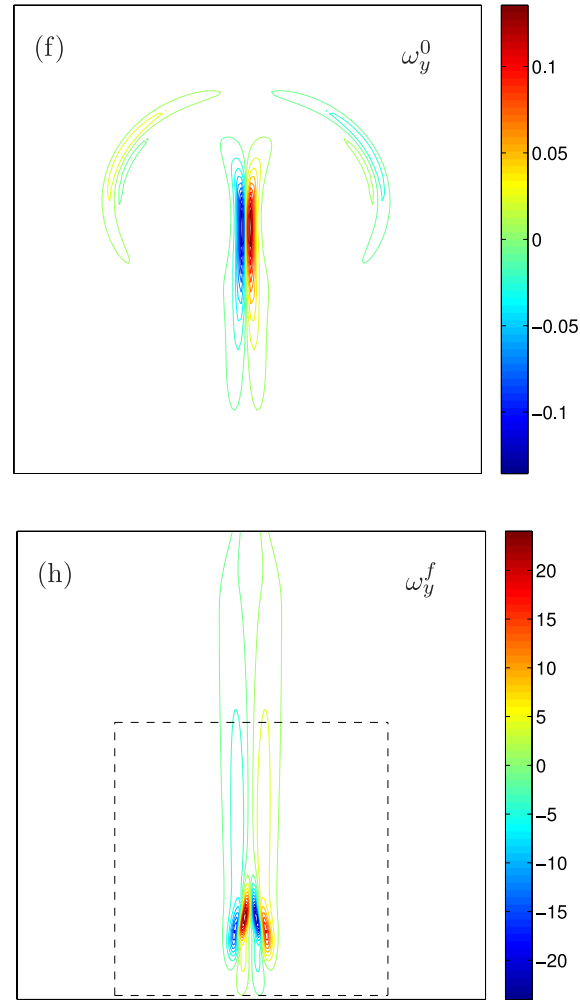

FIG. 17. Transient Crow type instability: symmetric optimal perturbations for the long-wavelength peaks $\left(k_{y}^{*} a_{0}=0.2\right)$ at $\tau=10$-same legend as Fig. 16 but at $\tau=10$, for (a)-(c) $F r=5$ and (a)-(c) $F r=2$, and the size of the domain shown is $3.5 b_{0} \times 3.5 b_{0}$ except for figure (h) where it is $6 b_{0} \times 6 b_{0}$ (the dashed box represents a domain of size $3.5 b_{0} \times 3.5 b_{0}$ for comparison with other figures).

of the optimal response consists of a tilted dipolar perturbation on each vortex which, as already discussed several times, induces a symmetric displacement of the vortices as a whole as in the Crow mechanism. These dipolar perturbations are slightly closer for $F r=2$ (Fig. 16(c)) when compared to $F r=\infty$ (Fig. 12(c)) since the stronger the stratification, the faster the vortices come closer. But the effect of the stratification is moderate since, for $F r=2$, the potential energy is less than $17 \%$ of the total final energy as shown in Table III of Appendix B.

For $F r=1$, the initial and final symmetric perturbations at $\tau=4$ are very different from larger Froude numbers since, for $F r=1$, the dipole is rapidly distorted while moving against the stratification. Still initial optimal perturbations are mainly inertial (Table III of Appendix B) and the mean growth rate is very similar to the unstratified case despite the much more complex optimal response structure with large axial vorticity perturbations located in the wake of the dipole (Fig. 17), the axial vorticity being still the largest component of the vorticity for both the initial and final perturbations.

$b$. Antisymmetric perturbations. The picture is radically different for the antisymmetric mode for which the mean growth rate strongly increases with decreasing Froude number. At $\tau=4$ and for $F r=2$, the mean growth rate of the antisymmetric mode equals the one of the symmetric modes and for $F r=1$, it supersedes the symmetric mode growth rate by $50 \%$ with a final optimal response still dominated by kinetic energy. At time horizon $\tau=10$, the antisymmetric optimal mean growth rate becomes larger than the symmetric one for $F r=2$. In the response, both for $F r=5$ and $F r=2$, the potential energy is now as important as the kinetic energy for both $\tau=4$ and $\tau=10$ (Table III). The explanation for the enhanced instability is lacking but comes from the destabilizing effect of density perturbations. 
For concision, the structure of the antisymmetric initial and final optimal perturbation is not shown here, since final perturbations resemble the symmetric one but with the opposite symmetry and the initial perturbations are close to the unstratified one with perturbations located on the contracting manifold of both stagnation points and not mainly on the lower one as for the symmetric case.

\section{Short wavelength dynamics}

The antisymmetric and symmetric transient growth rates present an extremum around $k_{y}^{*} a_{0}=$ 1.6 for all Froude numbers and time horizons $\tau=4$ and $\tau=10$. At $\tau=4$, the transient growth rate of antisymmetric perturbations at $k_{y}^{*} a_{0}=1.6$ doubles when going from $F r=\infty$ to $F r=1$ (Fig. 15(a)) and the same is observed in Fig. 15(b) for the symmetric mode. At $\tau=10$, the transient growth rate decreases for the symmetric mode (Fig. 15(d)) and for the antisymmetric mode between $F r=\infty$ and $F r=10$ only and even increases at $k_{y}^{*} a_{0}=1.6$ for $F r=5$ (Fig. 15(c)) but negligible for the symmetric mode (Fig. 15(d)). The increase of the transient growth rate when the Froude number decreases is coherent with the instantaneous linear elliptic instability since the smaller the Froude number the closer the vortices and the stronger the elliptic instability. Fig. 18 (respectively, Fig. 19) displays the axial vorticity of antisymmetric optimal perturbations for the wavenumber with the larger growth rate $k_{y}^{*} a_{0}=1.6$ for $\tau=4$ both for $F r=2$ and $F r=1$ (respectively, $k_{y}^{*} a_{0}=1.4$ for $\tau=10$ for $F r=5$ and $F r=2$ ). Symmetric perturbations, less unstable, are not shown but their optimal final perturbation structure is similar to the antisymmetric one whereas their initial perturbations resemble the one obtained for small wavenumber (Figs. 16(a), 17(a), and 17(b)) with perturbation only on the contracting manifold of the lower stagnation point.

$$
\tau=4
$$

$\operatorname{Fr}=2$
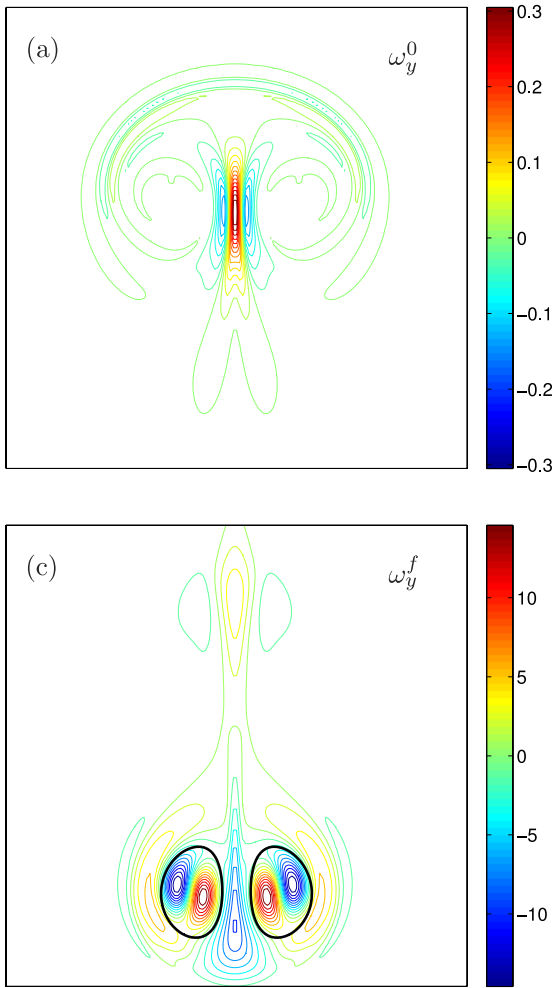

$F r=1$
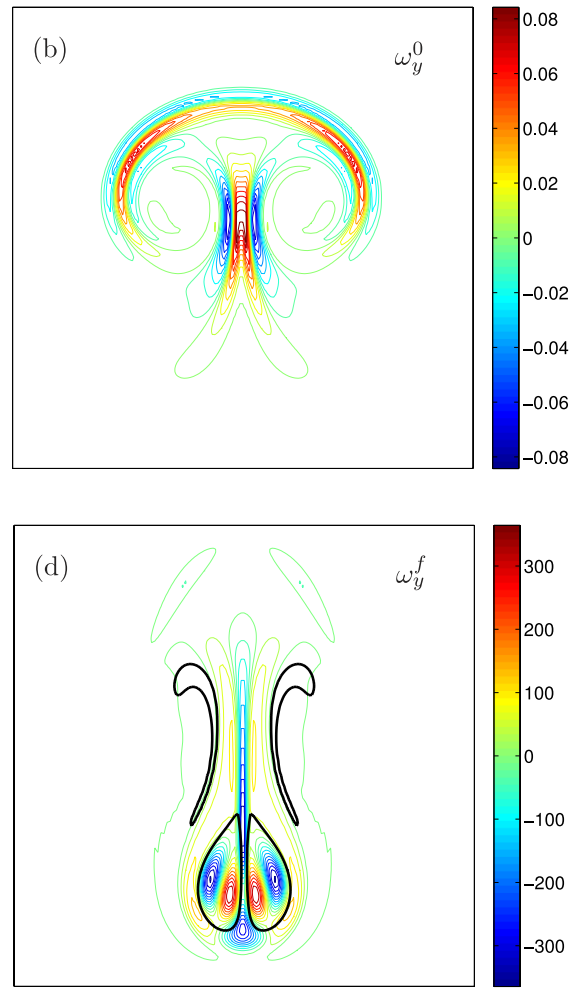

FIG. 18. Transient elliptic type instability: antisymmetric optimal perturbations for the short-wavelength $k_{y}^{*} a_{0}=1.6$ for $F r=2$ and $F r=1$ at $\tau=4$-same as Fig. 16 except that the perturbations are antisymmetric and $k_{y} a_{0}=1.6$. 
$\tau=10$

$\mathrm{Fr}=5$
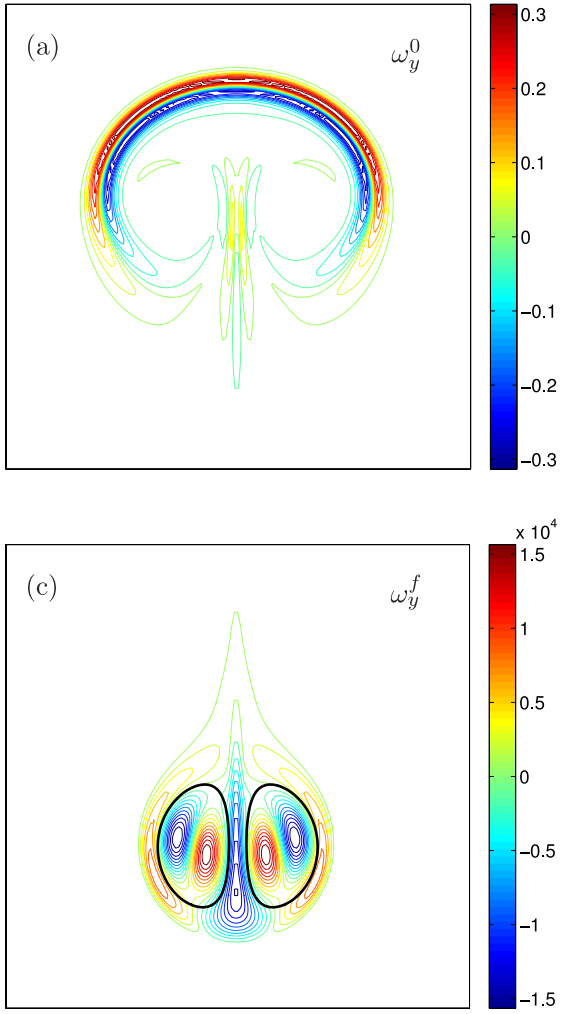

$F r=2$
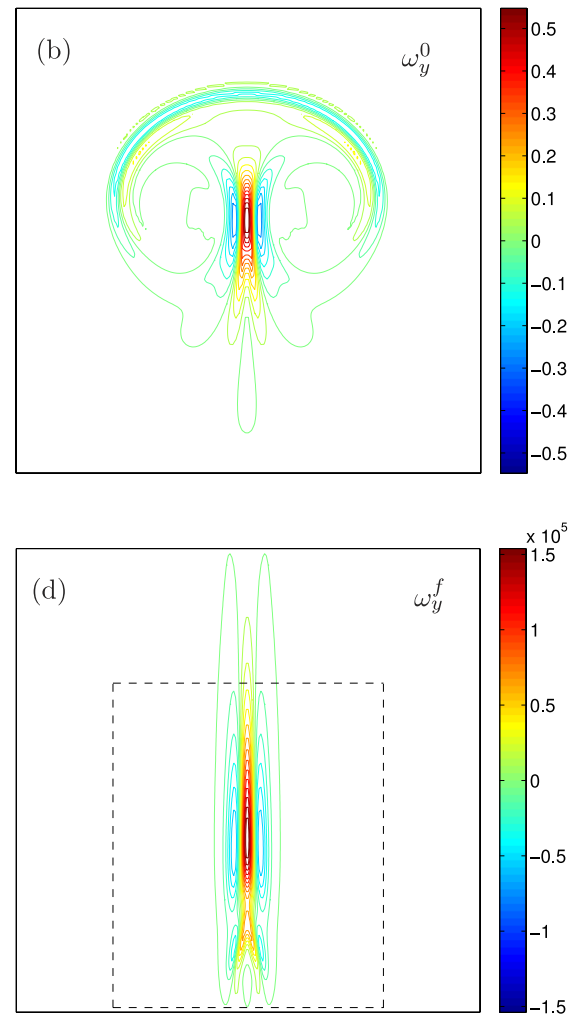

FIG. 19. Transient instability: antisymmetric optimal perturbations for the short-wavelength $k_{y}^{*} a_{0}=1.4$ at $\tau=10$ - same as Fig. 16 except that $\tau=10$, the perturbations are antisymmetric, $k_{y}^{*} a_{0}=1.4$ and (a)-(c) $F r=5$ and (a)-(c) $F r=2$. Note that the size of the domain shown is $5 b_{0} \times 5 b_{0}$ for (d) (larger than the domain of size $3.5 b_{0} \times 3.5 b_{0}$ represented by a dashed box for comparison with other figures).

For $\tau=4$ as Froude number decreases, the optimal initial perturbation evolves and its maximum axial vorticity migrates from the symmetry axis (Fig. 18(a)) to the contracting manifolds of the upper stagnation point of the base flow (Fig. 18(b)). The final optimal perturbation in each vortex resembles the elliptic mode with the characteristic dipolar perturbation of the axial vorticity concentrated inside the core of the vortex surrounded by opposite sign vorticity perturbation corresponding to the out of phase deformation between the core and periphery of the vortices. Surprisingly for $F r=1$ when vortices are nearly collapsed by time $\tau=4$ (see the thick lines representing the core of the vortices of Figs. 18 and 19), the final perturbations stay inside the cores surrounded by opposite sign vorticity in a structure reminiscent of the elliptic instability. On top of these perturbations similar to the elliptic modes located on the base flow vortices, large axial vorticity perturbations exist in the wake which develops behind the dipole (Figs. 18(c) and 18(d)).

At larger time $\tau=10$, the vortices have totally collapsed for $F r=2$, and the optimal response is then concentrated in the wake of the dipole where it is made of transverse (not shown) and axial (Fig. 19(d)) vorticity components with negligible vertical vorticity (not shown).

The optimal response shows the importance of the wake of the dipole that is not captured by the instantaneous linear stability analysis. As for the long wavelength dynamics discussed above, the density perturbations are associated with negligible potential energy compared to the kinetic energy of the perturbation for all Froude numbers computed (Table IV Appendix B) at time horizon $\tau=4$, but at time horizon $\tau=10$, equipartition is achieved both for $F r=2$ and 5. For $F r=5$, the vorticity of the optimal response is still reminiscent of the elliptic mode (Fig. 19), suggesting that 
density perturbations might not modify the instability mechanism and are generated by velocity perturbation.

\section{CONCLUSION}

The effects of the stratification on the two- and three-dimensional dynamics of a Lamb-Oseen vortex pair are investigated. The Froude numbers considered vary from $\infty$ to 1 .

The temporal evolution of the 2D flow has been computed using direct numerical simulations. As observed by Garten et al., ${ }^{7}$ the vorticity created by the baroclinic torque which appears around the dipole pushes the two vortices closer together, resulting in an increase of the deformation of each vortex core. At later stages, a wake develops behind the dipole. Due to the presence of the stratification, the dipole parameters (radius $a$, separation distance $b$, and circulation $\Gamma$ ) evolve with time. Temporal evolutions of these parameters once scaled by the Brunt-Väisälä frequency are nearly independent of the Froude number. The advection time of the dipole remains constant on a time scale of the order of the inverse of the Brunt-Väisälä frequency for all the stratifications whereas the ellipticity of the vortices, measured here by the ratio of their core size $a$ of their separation distance $b$, rapidly increases.

Both the stability analysis freezing the flow (i.e., using the quasi-steady approximation) and transient growth analysis on the time evolving base flow show, in presence of vertical stratification, that the growth of the perturbation is dominated by inertial mechanism associated with the development of Crow at long wavelength and elliptic modes at short wavelength for both symmetries. Up to time $N t^{*}=\mathrm{Fr}^{-1} t=4$ and for Froude numbers down to unity, the effect of the stratification is mainly to enhance the development of the elliptic instability by bringing the vortices closer together and therefore increasing their ellipticity confirming the mechanism proposed by Nomura et al. ${ }^{12}$ Even though inertial effects in the vortices dominate the dynamics, optimal response is not limited to the core of the vortices and affects also the wake of the dipole. In the present study, only order one ratio of the core to the distance of the vortices has been considered. Such vortices do not correspond to aircraft vortices that are more concentrated, still since we show that down to Froude number unity the dynamics is mainly led by the elliptic instability, therefore by inertial effects rather than gravity

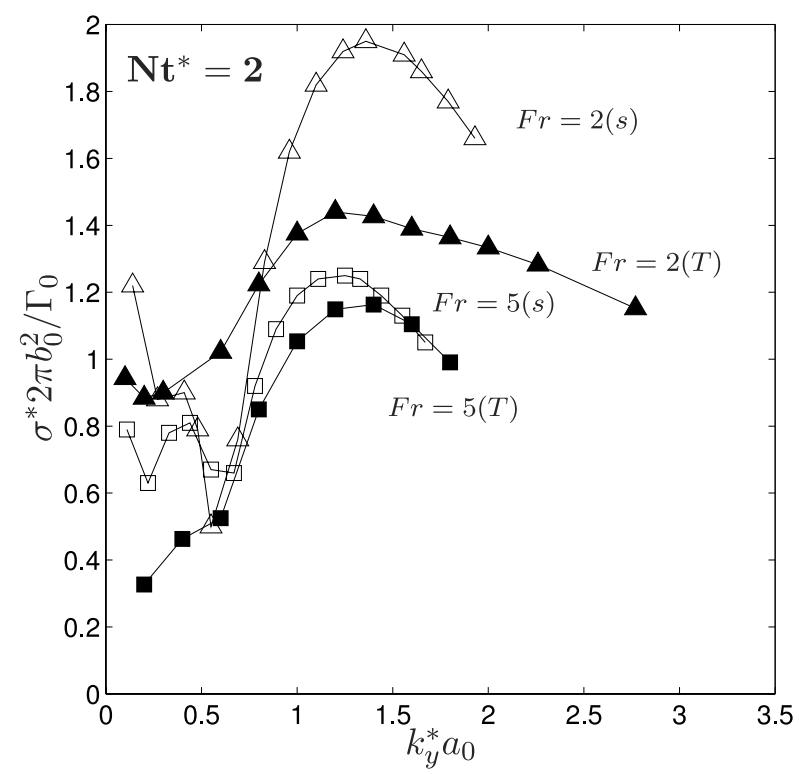

FIG. 20. Comparison between quasi-steady linear growth rate and optimal mean transient growth rate at $N t^{*}=\mathrm{Fr}^{-1} t=2$ for stratified flows - transient growth rate (closed symbols) and growth rates $\sigma^{*}$ (open symbols) from the quasi-steady linear stability analysis on the final frozen state of the antisymmetric modes as function of the axial wavenumber $k_{y}^{*}$ scaled with the initial values of the radius $a_{0}$, the separation distance $b_{0}$, and the circulation $\Gamma_{0}$ of the vortices at $N t^{*}=F r^{-1} t=2$ for $\operatorname{Re}_{\Gamma_{0}}=2400$ and for $F r=5(\square, \boldsymbol{\square})$ and $F r=2(\Delta, \Delta)$. 
effects, we may conjecture that the mechanism described in this study should be retrieved. However, the investigation of such aircraft trailing vortices requires very high resolution and deserves a thoughtful study out of the scope of the present paper before asserting such a conclusion.

For the short wave instability comparison between the linear stability (Sec. III) and optimal perturbations (Sec. IV), analysis is plotted in Fig. 20 for the antisymmetric case at $N t^{*}=2$ for $F r=5$ and $F r=2$ corresponding, respectively, to $\tau=10$ and $\tau=4$. The quasi-steady linear growth rates (s) are shown in Fig. 6(c) and the transient growth rates (T) in Figs. 15(a) and 15(c). For the less stratified flow $F r=5$, the results compare relatively well for the short-wavelength peak, while for the strongly stratified case $F r=2$, the two curves differ. Positions of the maximum are close ( $k_{y}^{*} a_{0}=1.2$ for optimal perturbations and $k_{y}^{*} a_{0}=1.36$ for quasi-steady analysis) but the corresponding transient growth rate for the optimal perturbations is lower by $30 \%$ than the growth rate computed with quasi-steady analysis. The peak is broader for the optimal perturbations indicating that the optimal perturbations are less selective than predicted by quasi-steady theory a property already observed and discussed for the unstratified case.

\section{APPENDIX A: VALIDATION OF THE DIRECT-ADJOINT TECHNIQUE WHICH TAKES INTO ACCOUNT THE EVOLUTION OF THE BASE FLOW}

In order to validate our method, the results of the new code that takes into account the evolution of the base flow have been compared to the former one where the base flow is frozen in the unstratified case. For the former code, the aspect ratio of the dipole of the base flow was $a / b=0.2$. Therefore, the optimal perturbations were computed for a dipole of initial aspect ratio $a_{0} / b_{0}=0.2$ for a Reynolds number $\operatorname{Re}_{\Gamma_{0}}=2000$ and for $F r=\infty$ at $\tau=1$. Between $t=0$ and $t=1$, the radius of the vortices has grown by $2 \%$ through viscous diffusion but the separation distance between the vortices and the circulation of the vortices has remained constant. The optimal perturbations at $\tau=t^{*} \Gamma^{*} / 2 \pi b^{* 2}=1$ on a dipole of aspect ratio $a / b=0.2$ computed with the former code for $R e_{\Gamma}=2000$ and for a homogeneous fluid, corresponding to case 1 of Table II, have first been compared to the optimal perturbations computed with the new code which takes into account the evolution of the base flow but the initial state, consisting in the elliptic counter-rotating vortices of aspect ratio $a_{0} / b_{0}=0.2$ in the reference frame of the dipole, was read at $t=0$ and frozen during the forward and backward integrations, corresponding to case 2 of Table II. The enstrophy of optimal initial perturbations (Figs. 21(a) and 21(c)) and optimal responses (Figs. 21(b) and 21(d)) is similar and the transient growth rates (cases 1 and 2 of Table II) are equal. Case 1 of Table II has then be compared to the optimal perturbations computed with the new code which takes into account the evolution of the base flow with initial state consisting of two circular Lamb-Oseen vortices of initial aspect ratio $a_{0} / b_{0}=0.2$, which is read every $10 \Delta t$ and computed every $\Delta t$ via a linear interpolation as mentioned in Sec. IV A; this corresponds to case 3 of Table II. The mean growth rate (case 3 of Table II) is $2 \%$ lower than case 1 , which is in agreement with the growth of the radius of the vortices through viscous diffusion: the position of the maximum is shifted to a shorter value and, consequently, the growth rate is smaller at $k_{y}^{*} a_{0}=2.26$ since it is not the maximum of the peak anymore.

The accuracy of the computations has been tested for several values of $n, n \Delta t$ being the rate at which the base flow is updated for the interpolation procedure: the same computation as case 3 is performed with the base flow read every $40 \Delta t$ (case 4 of Table II), 100 $\Delta t$ (case 5 of Table II),

TABLE II. Validation-computational accuracy of the growth rate $\sigma^{*} 2 \pi b_{0}^{2} / \Gamma_{0}$ for the antisymmetric case at $k_{y} a_{0}=2.26$ for $R e_{\Gamma}=2000$ and for $F r=\infty$ at $\tau=1$ (cases 1 to 5) and $F r=2$ at $\tau=4$ (cases 7, 8,9). The bold cases correspond to the values of $n$ chosen for the computation of optimal perturbations of Sec. IV.

\begin{tabular}{|c|c|c|c|c|c|c|c|c|c|}
\hline \multirow[b]{2}{*}{ Case } & \multicolumn{6}{|c|}{$F r=\infty$} & \multicolumn{3}{|c|}{$F r=2$} \\
\hline & 1 & 2 & 3 & 4 & 5 & 6 & 7 & 8 & 9 \\
\hline$n$ & 0 & 0 & 10 & 40 & 100 & 200 & 40 & 100 & 200 \\
\hline$\sigma^{*} 2 \pi b_{0}^{2} / \Gamma_{0}$ & 1.80 & 1.80 & 1.76 & 1.76 & 1.75 & 1.74 & 1.29 & 1.28 & 1.24 \\
\hline
\end{tabular}


(a)

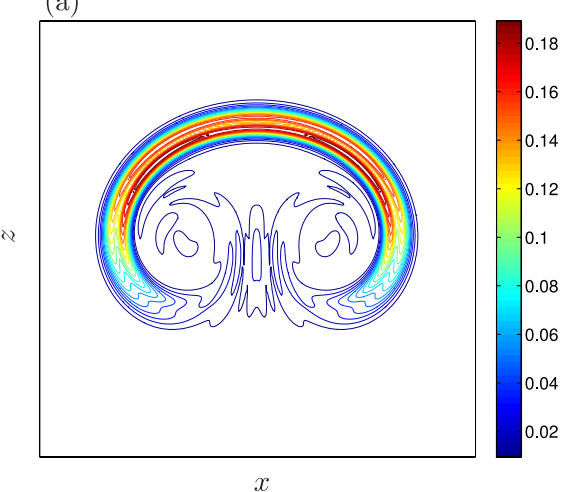

(c)

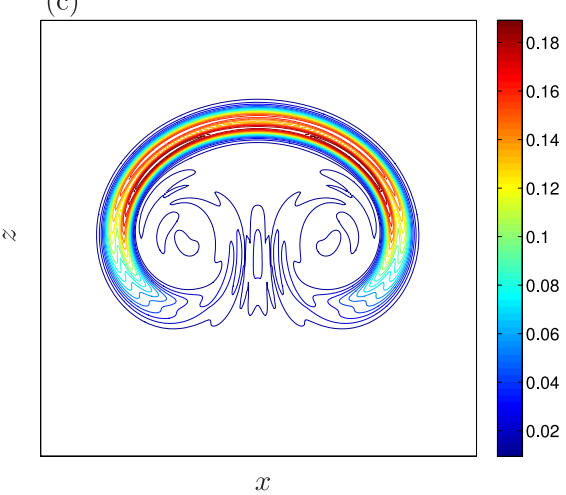

(e)

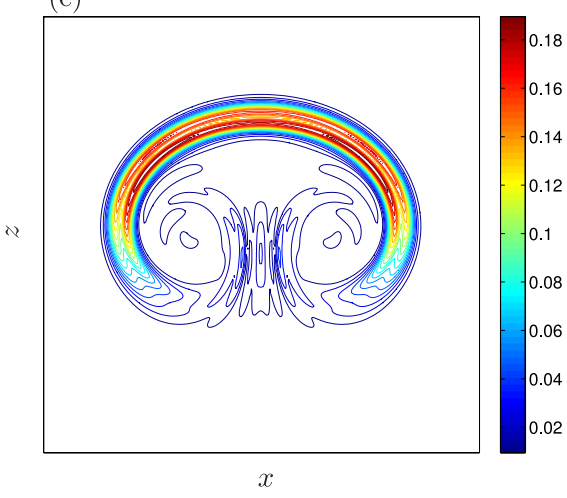

(b)

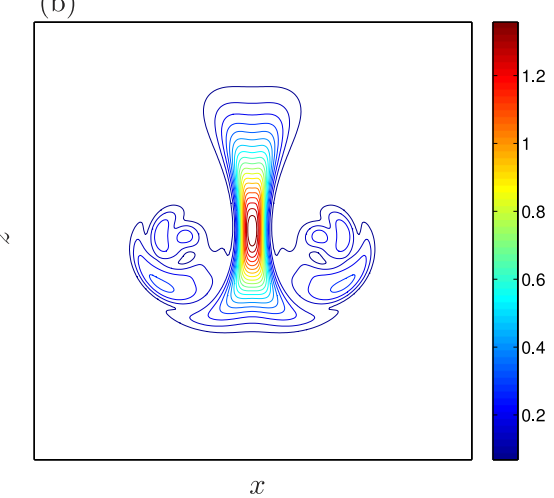

(d)

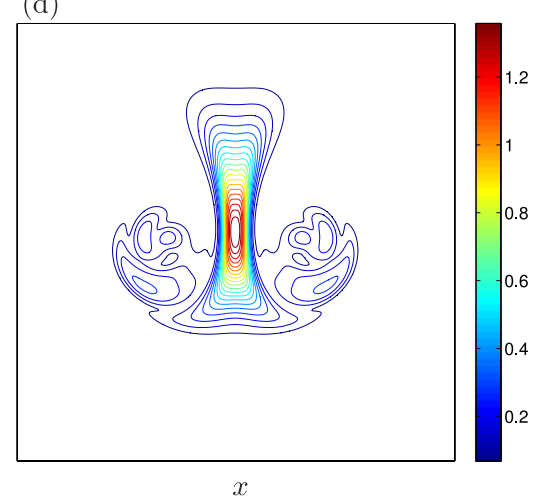

(f)

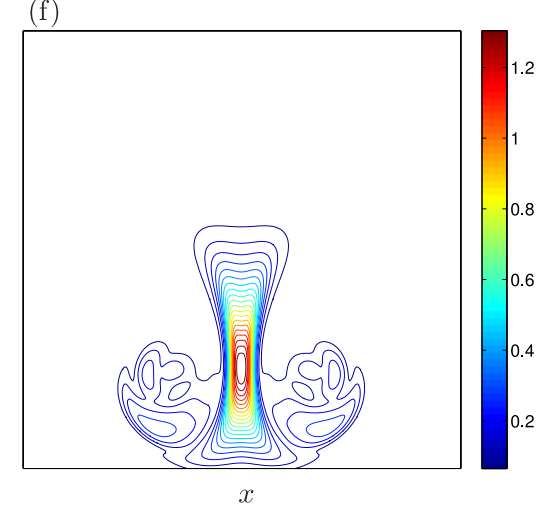

FIG. 21. Validation-enstrophy of (a), (c), and (e) the optimal perturbation and (b), (d), and (f) the optimal response of the antisymmetric case at the maximum of the elliptic instability band $k_{y} a_{0}=2.26$ for $R e_{\Gamma}=2000$ and $F r=\infty$ at $\tau=1$. (a) and (b) Former code (Donnadieu et al. ${ }^{13}$ ) which does not take into account the evolution of the base flow. (c) and (d) New code which takes into account the evolution of the base flow but with a frozen base flow with an aspect ratio $a / b=0.2$. (e) and (f) New code which takes into account the evolution of the base flow, which is read every $40 \Delta t$. The size of the domain shown is $3 b \times 3 b$ whereas the computation domain is $3 b \times 3 b$ for (a) and (b) and $6 b \times 6 b$ for (c)-(f).

and $200 \Delta t$ (case 6 of Table II). The optimal perturbations corresponding to case 4 of Table II are displayed in Figs. 21(e) and 21(f). The spatial distribution of the enstrophy of optimal initial perturbation and optimal response computed on this transiently and diffusing dipole (Figs. 21(e) and 21(f)) is similar to frozen case 1 (Figs. 21(a) and 21(b)) except that the optimal response is at a lower height since the base flow vortices have propagated downwards between $t=0$ and $t=1$. The growth rate of case 4 is equal to case 3, whereas it is lower by $0.5 \%$ for case 5 and by $1 \%$ for case 6 .

For the stratified flows, the base flow changes strongly between $t=0$ and final time horizon: on top of viscous diffusion, the buoyancy force deforms the vortices and baroclinic vorticity appears around and behind the dipole. Therefore, the accuracy of the computations has also been tested for 
the moderately stratified fluid $F r=2$ at $\tau=4$ for $k_{y}^{*} a_{0}=2.26$ (cases 7, 8, and 9 of Table II). The growth rate of case $7(n=40)$ is larger by $1 \%$ than case $8(n=100)$ and by $4 \%$ than case $9(n=200)$.

The value of $n$ has been chosen equal to 40 for $\tau=4$ and to 100 for $\tau=10$ which provides the best compromise between accuracy and use of memory.

\section{APPENDIX B: TABLE COLLECTING VALUES OF KINETIC AND POTENTIAL ENERGY OF OPTIMAL PERTURBATIONS}

TABLE III. Energy normed by the initial total energy at large scale $\mathrm{k}_{\mathrm{y}}^{*} \mathrm{a}_{0}=0.2, \mathrm{E}^{0}, \mathrm{E}^{\mathrm{f}}$ stand for the initial and final energy, the indices $c$ and $p$ for kinetic and potential energy.

\begin{tabular}{|c|c|c|c|c|c|c|c|}
\hline \multicolumn{8}{|c|}{ Antisymmetric } \\
\hline \multirow[b]{2}{*}{$\mathrm{Fr}$} & \multicolumn{4}{|c|}{$\tau=4$} & \multicolumn{3}{|c|}{$\tau=10$} \\
\hline & 10 & 5 & 2 & 1 & 10 & 5 & 2 \\
\hline $\mathrm{E}_{\mathrm{c}}^{0}$ & 1 & 1 & 0.997 & 0.964 & 1 & 1 & 0.994 \\
\hline $\mathrm{E}_{\mathrm{p}}^{0}$ & $1.365 \times 10^{-6}$ & $7.250 \times 10^{-5}$ & $3.086 \times 10^{-3}$ & $3.624 \times 10^{-2}$ & $3.29 \times 10^{-6}$ & $1.524 \times 10^{-4}$ & $6.080 \times 10^{-3}$ \\
\hline$E^{f}$ & $1.256 \times 10^{2}$ & $1.551 \times 10^{2}$ & $1.261 \times 10^{3}$ & $2.673 \times 10^{5}$ & $1.486 \times 10^{2}$ & $7.719 \times 10^{2}$ & $2.173 \times 10^{7}$ \\
\hline $\mathrm{E}_{\mathrm{c}}^{f}$ & $1.228 \times 10^{2}$ & $1.445 \times 10^{2}$ & $1.106 \times 10^{3}$ & $2.169 \times 10^{5}$ & $1.073 \times 10^{2}$ & $5.270 \times 10^{2}$ & $1.117 \times 10^{7}$ \\
\hline$E_{p}^{f}$ & 2.727 & 10.60 & $1.551 \times 10^{2}$ & $5.040 \times 10^{4}$ & $0.413 \times 10^{2}$ & $2.449 \times 10^{2}$ & $1.056 \times 10^{7}$ \\
\hline \multicolumn{8}{|c|}{ Symmetric } \\
\hline \multicolumn{5}{|c|}{$\tau=4$} & \multicolumn{3}{|c|}{$\tau=10$} \\
\hline Fr & 10 & 5 & 2 & 1 & 10 & 5 & 2 \\
\hline $\mathrm{E}_{\mathrm{c}}^{0}$ & 1 & 1 & 0.994 & 0.886 & 1 & 1 & 0.995 \\
\hline $\mathrm{E}_{\mathrm{p}}^{0}$ & $4.429 \times 10^{-7}$ & $2.765 \times 10^{-5}$ & $5.475 \times 10^{3}$ & 0.114 & $5.146 \times 10^{-7}$ & $2.970 \times 10^{-5}$ & $5.311 \times 10^{-3}$ \\
\hline$E^{f}$ & $3.947 \times 10^{3}$ & $4.112 \times 10^{3}$ & $5.680 \times 10^{3}$ & $1.016 \times 10^{4}$ & $1.663 \times 10^{6}$ & $4.389 \times 10^{6}$ & $6.222 \times 10^{5}$ \\
\hline$E_{c}^{f}$ & $3.913 \times 10^{3}$ & $3.974 \times 10^{3}$ & $4.715 \times 10^{3}$ & $7.450 \times 10^{3}$ & $1.414 \times 10^{6}$ & $2.189 \times 10^{6}$ & $2.778 \times 10^{5}$ \\
\hline$E_{p}^{f}$ & 33.95 & $1.383 \times 10^{2}$ & $9.648 \times 10^{2}$ & $2.713 \times 10^{3}$ & $2.490 \times 10^{5}$ & $2.200 \times 10^{6}$ & $3.444 \times 10^{5}$ \\
\hline
\end{tabular}

TABLE IV. Energy normed by the initial total energy at $k_{y}^{*} a_{0}=1.6$.

\begin{tabular}{|c|c|c|c|c|c|c|c|}
\hline \multicolumn{8}{|c|}{ Antisymmetric } \\
\hline \multirow[b]{2}{*}{$\mathrm{Fr}$} & \multicolumn{4}{|c|}{$\tau=4$} & \multicolumn{3}{|c|}{$\tau=10$} \\
\hline & 10 & 5 & 2 & 1 & 10 & 5 & 2 \\
\hline $\mathrm{E}_{\mathrm{c}}^{0}$ & 1 & 1 & 0.999 & 0.938 & 1 & 1 & 0.999 \\
\hline $\mathrm{E}_{\mathrm{p}}^{0}$ & $3.702 \times 10^{-7}$ & $2.743 \times 10^{-5}$ & $1.068 \times 10^{-3}$ & $6.179 \times 10^{-2}$ & $4.031 \times 10^{-7}$ & $2.631 \times 10^{-5}$ & $1.074 \times 10^{-3}$ \\
\hline$E^{f}$ & $4.166 \times 10^{3}$ & $3.377 \times 10^{3}$ & $6.805 \times 10^{4}$ & $4.238 \times 10^{7}$ & $5.281 \times 10^{7}$ & $4.158 \times 10^{9}$ & $3.408 \times 10^{11}$ \\
\hline$E_{c}^{f}$ & $4.152 \times 10^{3}$ & $3.350 \times 10^{3}$ & $6.019 \times 10^{4}$ & $3.663 \times 10^{7}$ & $4.358 \times 10^{7}$ & $2.036 \times 10^{9}$ & $2.006 \times 10^{11}$ \\
\hline$E_{p}^{f}$ & 13.30 & 26.53 & $7.854 \times 10^{3}$ & $5.743 \times 10^{6}$ & $9.228 \times 10^{6}$ & $2.122 \times 10^{9}$ & $1.402 \times 10^{11}$ \\
\hline \multicolumn{8}{|c|}{ Symmetric } \\
\hline \multicolumn{5}{|c|}{$\tau=4$} & \multicolumn{3}{|c|}{$\tau=10$} \\
\hline $\mathrm{Fr}$ & 10 & 5 & 2 & 1 & 10 & 5 & 2 \\
\hline $\mathrm{E}_{\mathrm{c}}^{0}$ & 1 & 1 & 0.997 & 0.954 & 1 & 1 & 0.993 \\
\hline $\mathrm{E}_{\mathrm{p}}^{0}$ & $6.759 \times 10^{-7}$ & $4.463 \times 10^{-5}$ & $3.115 \times 10^{-3}$ & $4.597 \times 10^{-2}$ & $7.331 \times 10^{-7}$ & $5.536 \times 10^{-5}$ & $6.550 \times 10^{-3}$ \\
\hline$E^{f}$ & $1.178 \times 10^{3}$ & $1.257 \times 10^{3}$ & $1.185 \times 10^{4}$ & $2.010 \times 10^{6}$ & $2.792 \times 10^{6}$ & $4.972 \times 10^{7}$ & $3.350 \times 10^{7}$ \\
\hline$E_{c}^{f}$ & $1.124 \times 10^{3}$ & $9.835 \times 10^{2}$ & $8.469 \times 10^{3}$ & $1.663 \times 10^{6}$ & $2.322 \times 10^{6}$ & $1.739 \times 10^{7}$ & $1.596 \times 10^{7}$ \\
\hline$E_{p}^{f}$ & 53.62 & $2.740 \times 10^{2}$ & $3.377 \times 10^{3}$ & $3.478 \times 10^{5}$ & $4.700 \times 10^{5}$ & $3.233 \times 10^{7}$ & $1.754 \times 10^{7}$ \\
\hline
\end{tabular}


${ }^{1}$ S. C. Crow, "Stability theory for a pair of trailing vortices," AIAA J. 8(12), 2172-2179 (1970).

${ }^{2}$ D. W. Moore and P. G. Saffman, "The instability of a straight filament in a strain field," Proc. R. Soc. A 346, 413-425 (1975).

${ }^{3}$ C.-Y. Tsai and S. E. Widnall, "The stability of short waves on a straight vortex filament in a weak externally imposed strain field," J. Fluid Mech. 73, 721-733 (1976).

${ }^{4}$ R. S. Scorer and L. J. Davenport, “Contrails and aircraft downwash,” J. Fluid Mech. 43, 451-464 (1970).

${ }^{5}$ F. M. Hill, "A numerical study of the descent of a vortex pair in stably stratified atmosphere," J. Fluid Mech. 71, 1-13 (1975).

${ }^{6}$ P. R. Spalart, "On the motion of laminar wing wakes in a stratified fluid," J. Fluid Mech. 327, 139-160 (1996).

${ }^{7}$ J. F. Garten, S. Arendt, D. C. Fritts, and J. Werne, "Dynamics of counter-rotating vortex pairs in stratified and shears environments," J. Fluid Mech. 361, 189-236 (1998).

${ }^{8}$ T. Sarpkaya, "Trailing vortices in homogeneous and density-stratified media," J. Fluid Mech. 136, 85-109 (1983).

${ }^{9}$ D. P. Delisi and R. E. Robins, "Short-scale instabilities in trailing wake vortices in a stratified fluid," AIAA J. 38, 1916-1923 (2000).

${ }^{10}$ R. E. Robins and D. P. Delisi, "Numerical simulation of three-dimensional trailing vortex evolution in stratified fluid," AIAA J. 36, 981-985 (1998).

${ }^{11}$ J. F. Garten, J. Werne, D. C. Fritts, and S. Arendt, "Direct numerical simulations of the Crow instability and subsequent vortex reconnection on a stratified fluid," J. Fluid Mech. 426, 1-45 (2001).

${ }^{12}$ K. K. Nomura, H. Tsutsui, D. Mahoney, and J. W. Rottman, "Short-wavelength instability and decay of a vortex pair in stratified fluid,” J. Fluid Mech. 553, 283-322 (2006).

${ }^{13}$ C. Donnadieu, S. Ortiz, J.-M. Chomaz, and P. Billant, "Three-dimensional instabilities and transient growth of a counterrotating vortex pair," Phys. Fluids 21(9), 094102 (2009).

${ }^{14}$ I. Delbende, J.-M. Chomaz, and P. Huerre, "Absolute/convective instabilities in the Batchelor vortex: A numerical study of the linear impulse response," J. Fluid Mech. 355, 229-254 (1998).

15 G. K. Batchelor, An Introduction to Fluid Dynamics (Cambridge University Press, 1967).

${ }^{16}$ P. G. Saffman, "The motion of the vortex pair in a stratified atmosphere," Stud. Appl. Math. 51(2), 107-119 (1972).

${ }^{17}$ D. P. Delisi, G. Greene, R. E. Robins, D. Vicroy, and F. Wang, "Aircraft wake vortex core size measurements," in 21st AIAA Applied Aerodynamics Conference, Orlando Florida (American Institute of Aeronautics and Astronautics, 2003), p. 3811.

${ }^{18}$ C. P. Caulfield and R. Peltier, "Three dimensionalization of the stratified mixing layer," Phys. Fluids 12(6), 3803-3805 (1994).

${ }^{19}$ S. Le Dizès and F. Laporte, "Theoretical predictions for the elliptical instability in a two-vortex flow," J. Fluid Mech. 471, 120-130 (2002).

${ }^{20}$ D. Sipp and L. Jacquin, "Widnall instabilities in vortex pairs,” Phys. Fluids 15, 1861-1874 (2003).

${ }^{21}$ P. Luchini, "Reynolds-number-independant instability of the boundary layer over a flat surface: Optimal perturbations," J. Fluid Mech. 404, 289-309 (2000).

${ }^{22}$ P. Corbett and A. Bottaro, "Optimal perturbations for boundary layers subject to stream-wise pressure gradient," Phys. Fluids 12, 120-130 (2000).

${ }^{23}$ E. L. Ince, Ordinary Differential Equations (Cambridge University Press, 1944).

${ }^{24}$ S. Ortiz and J.-M. Chomaz, "Transient growth of secondary instabilities in parallel wakes: Anti lift-up mechanism and hyperbolic instability," Phys. Fluids 23(11), 11416 (2011).

${ }^{25}$ V. Brion, D. Sipp, and L. Jacquin, "Optimal amplification of the Crow instability," Phys. Fluids 19, 111703 (2007).

${ }^{26}$ D. Sipp, L. Jacquin, and C. Cossu, "Self-adaptation and viscous selection in concentrated 2D vortex dipole," Phys. Fluids 12, 245-248 (1999).

${ }^{27}$ T. Leweke and C. Willamson, "Cooperative elliptic instability of a vortex pair," J. Fluid Mech. 360, 85-119 (1998). 